Review,

\title{
Apoptosis in type 2 diabetes, can it be prevented?
}

\section{Agnieszka Kilanowska ${ }^{1 *}$, Agnieszka Ziółkowska ${ }^{1}$}

1 Affiliation 1; University of Zielona Gora, Collegium Medicum, Department of Anatomy and Histology; Zyty 28

* Correspondence: a.kilanowska@cm.uz.zgora.pl;

\begin{abstract}
Diabetes mellitus is a heterogeneous disease of complex etiology and pathogenesis. Hyperglycemia leads to many serious complications, but also directly initiates the process of $\beta$ cell apoptosis. A potential strategy for the preservation of pancreatic $\beta$ cells in diabetes may be to inhibit the implementation of pro-apoptotic pathways or to enhance the action of pancreatic protective factors. The HIPPO signaling pathway is proposed and selected as a target to manipulate the activity of its core proteins in therapy - basic research. MST1 and LATS2 as major upstream signaling kinases of the Hippo pathway are considered as target candidates for pharmacologically induced tissue regeneration and inhibition of apoptosis. Manipulating the activity of components of the HiPPO pathway offers a wide range of possibilities, and thus is a potential tool in the treatment of diabetes and the regeneration of $\beta$ cells. Therefore, it is important to fully understand the processes involved in apoptosis in diabetic states and to fully characterize the role of this pathway in diabetes. Therapy consisting in slowing down or stopping the mechanisms of apoptosis may be an important direction of diabetes treatment shortly.
\end{abstract}

Keywords: type 2 diabates; Hyppo pathway, MST, LATS. YAP, apoptosis, caspase, $\beta$ cells, pancreatic islets,

\section{Introduction}

Diabetes mellitus is a heterogeneous disorder with multiple etiologies $[1,2]$. The most popular, in adults is type 2, a chronic metabolic illness with increasing medical and financial cost [3,4]. The incidence of diabetes in 2015 was $8.8 \%$, and in 2040 it will probably increase to 10.4\% [5]. Its most characteristic symptom - high glucose level being the result of insufficient insulin for the organism needs [6]. Symptoms, on the other hand, are a consequence of genetic and/or lifestyle factors that are generally a consequence of obesity [7,8]. The $\beta$ cell dysfunction and worsening insulin resistance over time lead to deterioration of glycemic control and, as a result, more intensive pharmacotherapy $[9,10]$. Over time, this usually manifests itself as complete endogenous islet inability resulting from loss of $\beta$ cells $[10,11]$ to which contribute also the mechanisms leading to the impairment of proliferation and dedifferentiation [13,14]. In type 2 diabetes, metabolic stress can induce pro-inflammatory cytokines: tumor necrosis factor- $\alpha$ (TNF- $\alpha$ ) and interleukin-6 (IL-6), and the consequence of complex processes leading to apoptotic death of pancreatic $\beta$ cells $[15,16]$. Glucotoxicity, lipotoxicity [17-20], endoplasmatic reticulum (ER) stress [21,22], oxidative stress [23,24], islet amyloid polypeptide (IAPP) [25] and inflammation $[15,26]$ are believed stimuli to be responsible for the loss of pancreatic mass and lead to progressive pancreatic $\beta$-cell failure in type 2 diabetes $[19,27,28]$. Destabilization of the endoplasmic reticulerum (ER) [29] poses a huge threat to its function in the context of the secretory pathway, which depends on the efficiency of translation and folding of extracellular proteins [30,31]. The inability to function by the ER is related to the mobilization of calcium from the ER pool in the $\beta$ cells of the pancreas [32]. Saturated fatty acids (e.g. palmitate) have been shown to contribute to apoptosis through the induction of reactive oxygen species (ROS) and the ER stress pathway [33], a similar effect was also observed in chronic hyperglycemia [34]. Induced ER stress is manifested by an imbalance between the ability to fold proteins in relation to the needs of the cell, leading to disturbances in homeostasis [35]. The functional disproportion causes the deposition of unfolded or misfolded proteins in the lumen of the ER [36]. Unfolded protein response (UPR) is an adaptive mechanism to restore homesotase [34]. It consists, inter 
alia, on increasing the functional capacity of organelles by transcriptional regulation of folding enzymes and chaperones and increasing the size of the ER. However, it is additionally characterized by a reduction in the influx of newly synthesized proteins by suppressing protein translation by phosphorylation of the eukaryotic translation initiation factor 2 (eIF2) as well as mRNA degradation [42]. The endoplasmic reticulum stress transducers present in the membrane oversee this process through the two luminal domains. One of them is involved in unfolded protein recognition. The second cytoplasmic domain sends signals to the cytosol and nucleus if the stabilization measures taken are ineffective [37]. If this mechanism fails to meet expectations, the same communication system is used to begin apoptosis pathway [36]. The apoptotic pathway involves two protein families, including the Bcl-2 protein family and caspases [38] which is the basis for distinguishing two pathways of apoptosis [39]. The scientific literature emphasizes the importance of two major apoptotic pathways. First called as "external" pathway (related to Fas or TNF death receptors (TNFR)) [25]. The second one is defined as the internal pathway (associated with the mitochondria) and carried out by the involved proteins from the Bcl-2 family $[29,40]$. Therefore, there are usually two main pathways: "death receptors" related and mitochondrial dependent [41-43]. The first of them is activated after the ligation of death receptors (e.g.: CD9, TNFR) on the cell surface. Expression of Fas in $\beta$ cells (external apoptotic pathway), is induced by cytokines and tumor necrosis factor alpha (TNF- $\alpha$ ), initiating the activation of the transcription factor, nuclear factor $\kappa b$, and signal transducer and activator of transcription 1 (STAT-1) and [44] leading in the consequences to the activation of caspases, proteins that implement the effector mechanism [45]. Caspases exist as zymogens in the soluble cytoplasm, endoplasmic reticulum (ER), mitochondrial intermembrane space, and nuclear matrix [46]. The Fas pathway initiates the formation of a multi-protein signaling complex causing death, a pathway controlled by caspases. The formation of a series of proteins of a signaling complex that induces death activates the apical caspase, procaspase-8, and the dynamic course of the apotposis process is further influenced by caspase 3 [44]. The second of the distinguished mechanisms emphasizes the role of mitochondrial processes. A characteristic feature of the mitochondrial apoptosis mechanism is the release of cytochrome c into the cytoplasm from the mitochondria. The effect is the activation of initiator caspase-9, resulting in the cleavage of executive caspases-3/7 [47]. Proteins from the Bcl-2 (an acronym for B-cell lymphoma 2 gene) family are involved in the integrity of mitochondria and are a complex group of proteins [48,49]. In the complex family of Blc-2 proteins, there are 3 groups: pro-apoptotic BH multidomain proteins (BAK, BAX, BOK), pro-survival proteins (A1 / BFL1, BCL-2, BCL-XL, BCL-W, MCL-1), and pro-apoptotic proteins with BCL-2 3 (BH3) homology (BAD, BID, BIM, BLK, BMF, DP5, NOXA, PUMA [40,48,50,51]. A dual adaptive anti-inflammatory / pro-apoptotic role of Bcl6 in $\beta$ cells has also been demonstrated [51]. The role of anti-apoptotic proteins is to weaken the internal death signaling associated with blocking the recruitment of pro-apoptotic members to the mitochondria [52]. The action of unfavorable factors on pancreatic $\beta$ cells, including, for example, long-term hyperglycemia disrupts the balance of the pro-apoptotic caspase family and anti-apoptotic Bcl proteins towards apoptosis, thus leading to the loss and depletion of islet cells [46]. Apoptosis as programmed cell death $[53,54]$ is a complex process characterized by cell shrinkage and chromatin condensation, but these preserve the integrity of the cell membrane [55]. Its biochemical features are revealed by the activation of specific cysteine proteases, caspases and the fragmentation of internucleosomal DNA [56,57]. DNA damage contributes to the activation of poly-ADP-ribose polymerase (PARP). Over-activation of PARP in $\beta$ cells contributes to the energy crisis by depleting ATP and results in cell death [58].

The need to modify the therapeutic procedure is a strong argument in favor of broadening the spectrum of observation of molecular processes and the possibility of breaking away from the usual procedures. Changes in the treatment of type 2 diabetes, at the hypothesis level, should effectively inhibit the loss of insulin-secreting cells while restoring their full functionality. Current therapies for treating type 2 diabetes only alleviate symptoms without ruling out the causes. The above assumptions require a complete analysis of the signal transduction network occurring in the 
process related to the loss of $\beta$ cells - apoptosis. Because its intense course ultimately results not only in the loss of pancreatic islet mass, but is also associated with morphological changes, including cell rounding or vesicle formation, and also by chromatin condensation [14,57]. Although the mechanism of apoptosis has been identified on many levels (triggering, initiating signaling cascades, and influencing the expression of apoptotic genes), but still some issues need to be clarified [59]. Intensive research in recent years shows promising prognosis and possibly a therapeutic footing in the Hippo pathway. It was proved that the general components and functions identified in Hippo pathway are involved in regulating organ size by maintaining a balance between apoptosis, proliferation and cell/tissue regeneration [60,61]. Identification of those signaling pathways on which the functioning and survival of pancreatic $\beta$ cells depends is a prerequisite for a full understanding of the mechanisms related to dysfunction and will be the basis for introducing new solutions in therapy [59]. The final number of cells in an organ is the result of a balanced act of cell proliferation and cell death (apoptosis) [62] and is dependent on the course of the Hippo pathway [63]. The aim of this review is to delineate the role of the Hippo signaling pathway in pancreatic $\beta$-cell apoptosis in diabetes, and the possibilities it presents in eliminating the consequences of diabetes in both cellular and systemic terms.

\section{The Hippo pathway - how it exacly works?}

The Hippo pathway is a complex communication network whose signals are modulated by numerous kinases/phosphatases and adapter proteins. The versatility and sensitivity of the mechanism causes its core to cross-react with other pathways to ensure adequate responses to exogenous and endogenous stimuli [64]. Its versatility is the basis for recognizing its position as a central regulator of organ size control, embryo development through the regulation of cells: proliferation, apoptosis and differentiation, in other words, maintaining tissue homeostasis [65]. Hippo pathway was first revealed in Drosophila melanogaster and named after Hippo kinase [66]. The components of this pathway, which has been confirmed many times, are evolutionarily conserved [67] and, importantly, preserved in all mammals [68]. The core of the mammalian Hippo pathway comprises mammalian sterile 20-like protein kinase 1 and 2 (MST1/MST2), two adaptor proteins Salvador (Sav1 scaffold protein) and MOB1A or MOB1B, Warts (Wts/large tumor suppressor 1 (LATS1) and LATS2 in mammals), two Yorkie homologs Yes-related protein (YAP in mammals) and transcription co-activator from PDZ binding motif (TAZ) and transcription factor TEA 1 (TEAD1) to TEAD4 (Figure 1) [57,69].

The course of this trail depends on the activity of its own components, creating conditions for observing its operation in two variants: when the Hippo pathway is on or off. In the first half MST1/2 kinase complexes with the adaptive protein Sav1 which are involved in the capture and phosphorylation of the NDR family kinases LATS1 LATS1/2. Activated LATS1/2 by phosphorylation and binding of MOB1A/B phosphorylates and inactivates major effectors of the Hippo pathway, including the Yes 1 (YAP) protein-related transcription coactivators and the PDZ binding motif transcription coactivator (TAZ). The mechanism of action of MST1/2 and LATS1/2 is an example of a negative regulation of YAP and TAZ activity by promoting their retention and/or degradation in the cytoplasm [59]. Phosphorylated YAP and TAZ is recognized by 14-3-3 protein which sequestered (YAP/TAZ) to the cytoplasm or degraded in a ubiquitin-proteasome dependent manner [70] which results in silencing (induced by the Hippo pathway) of gene transcription [71] the result is a reduction in the expression of YAP-dependent target genes, including anti-apoptotic and pro-survival genes [72]. It has been disclosed that genetic defects in the performance of the Hippo pathway in mice generate sustained tissue growth and can lead to cancer [73]. Conversely, when the Hippo pathway is off, its MST1/2 and LATS1/2 kinases are inactive. Unphosphorylated YAP and TAZ migrate to the nucleus. There they initiate the transcription and expression of genes responsible for proliferation, differentiation and apoptosis by interacting with TEAD1-4 and other transcription factors [66]. In studies where the Hippo pathway has been shown to be inactive (ie turned off), the transcriptional YAP and TAZ coactivators are hypophosphorylated and 
therefore translocate to the nucleus and induce their gene expression programs. It has been proven that many pathways converge in the YAP and TAZ registers, using their balancing in the nuclear/cytoplasmic region, which in turn translates into their transcriptional activity [74]. They work by recruiting transcription machinery for selected transcription factors, the best known of which is the TEAD family [71]. It is therefore considered responsible for the general phenotypes controlled by Hippo signaling.

The overall Hippo signal path is complex. It is now clear that both YAP1/TAZ, MST1/2 and LATS1/2 are regulated by other molecules. Regardless, the MST-LATS-YAP1 / TAZ-TEAD axis of interaction is the core of the mammalian Hippo pathway and is referred to as the canonical Hippo pathway [75].

\section{The mechanism of apoptosis in diabetes type 2 and role of Hippo pathway}

Many studies emphasize that pancreatic $\beta$ cells are extremely sensitive to oxidative stress due to the low level of antioxidant enzymes (catalase, glutathione peroxidase and superoxide dismutase) [23]. The involvement of the Hippo pathway in the regulation of the apoptotic process resulting from the metabolic changes associated with type 2 diabetes has been documented on the basis of the contribution of its individual elements, including MST1 [76], Merlin [77], LATS2 [78,79] and YAP [77,80].

The regulation and physiological functions of the kinases that make up the core of the Hippo pathway in mammalian cells are not fully understood. It was confirmed that apoptosis activated by various extracellular stimuli promotes apoptosis of cells by MST1/MST2, and caspase-catalyzed cleavage generates a highly active catalytic fragment, which is also associated with the process of progammed death [81]. The modulation of several signaling pathways and cellular organization dependent on the Hippo core kinases, especially in the area of cell polarity and the actin cytoskeleton [66]. MST or its truncated form induce morphological changes in cells, some features of which correspond to the morphology of apoptotic cells in vitro: rounding, detachment from the substrate, or fragmentation and condensation of the nucleus [76]. Moreover, overexpression of these kinases has been shown to induce apoptosis in a variety of cellular environments that are associated with the activation of stress-related pathways [82]. 


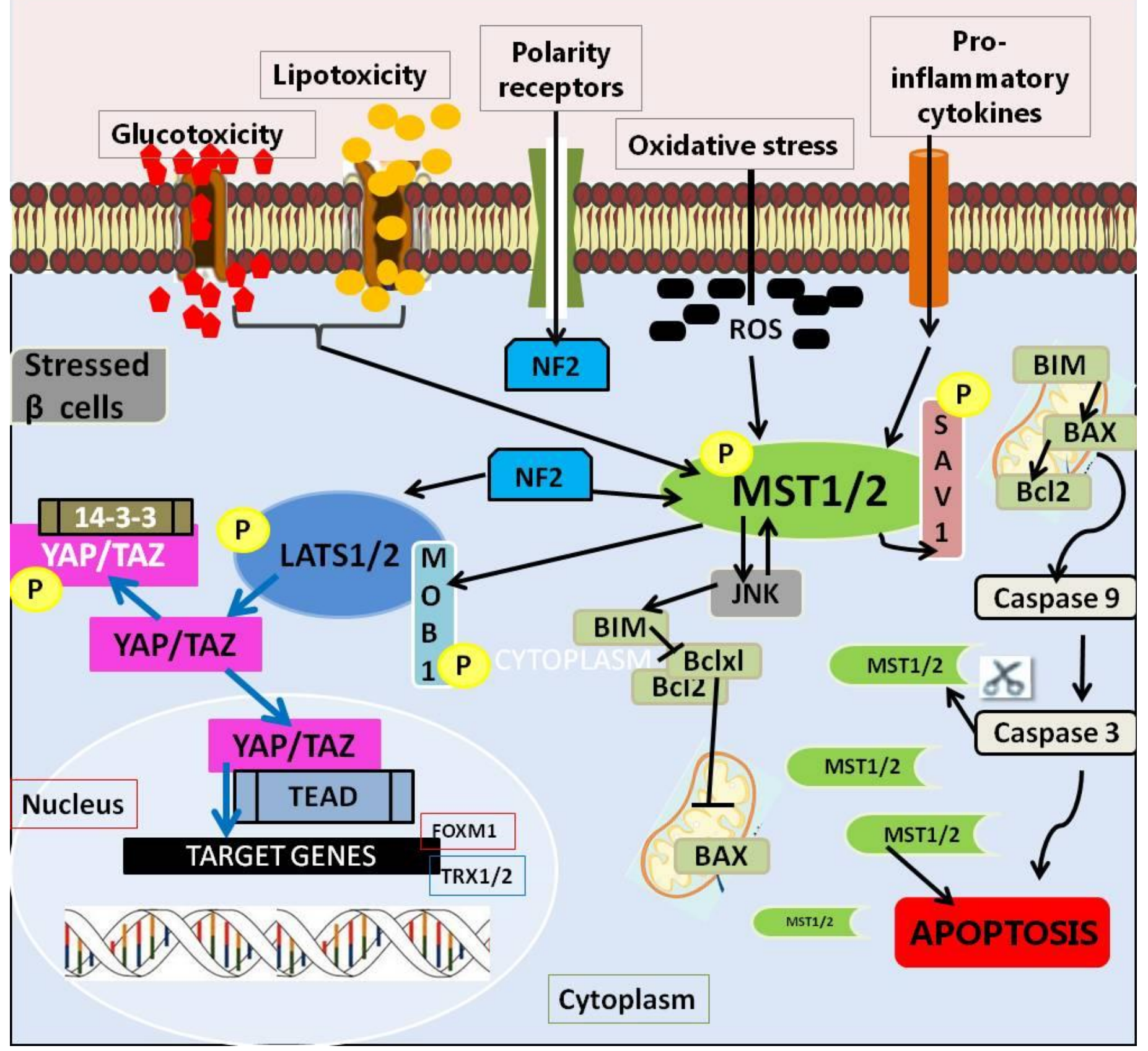

Figure 1. The Hippo pathwey in pancreatic $\beta$ cells.

\subsubsection{Upstream Regulators of Hippo Pathway}

\subsubsection{Merlin (neurofibromin 2, Nf2)}

The tumor suppressor gene Nf2, encodes the protein 4.1, Ezrin, Radixin, Moesin (FERM) domain-containing protein Merlin [85]. NF2 protein is a highly conserved in mammals and regulates the Hippo signaling pathway through MST1/MST1 and LATS1/LATS2 in $\beta$ cells (Figure 1) [86,87]. It acts as an initial activator of the HIPPO pathway, thus exerting a real influence on the regulation of cell growth and proliferation [85]. Two mechanisms of the initiation of HIPPO signaling by Merlin have been described. One is to initiate the pathway with MST-Sav activation followed by MST1/2 dependent LATS1/2 phosphorylation. The second one starts with the recruitment of LATS1/2 to the membrane [88]. Merlin silencing, especially in diabedogenic conditions (glucotoxic, glycolipotoxic and pro-inflammatory), brings many benefits to beta cells, protecting them against apoptosis. Moreover, both diabetogenic 
conditions and the introduced changes related to the loss of Merlin function in pancreatic $\beta$ cells reduced only the phosphorylation of LATS1/2, but not of MST1/2 [77,87].

\subsubsection{Protein RASS}

It has been disclosed that both MST1 and MST2 can form a constitutive homodimer via the SARAH domain, the autophosphorylation of the activation loop (T183 for Mst1 and T180 for Mst2) is the basis of kinase activation [64]. MST has been shown to interact with members of the Ras family of association domains of the MST/Hippo pathway (RASSF). The RASSF family of proteins consists of two subclasses, C-RASSF (RASSF1-6) and N-RASSF (RASSF7-10) [83] and carry a common domain of the Ras-association [84]. C-RASSF as regulatory proteins are characterized by a C-terminal coiled-coil motif - SARAH domain [82], same as Sav1 [83]. Briefly, a heterodimer is formed from the Mst1/2 domain of SARAH together with RASSF's SARAH [84] while a heterotetramer is formed from Sav1 SARAH. The Sav1 junction and the RASSFs binding to Mst1/2 are mutually exclusive [83]. It is through this SARAH domain that a set of RASSF effectors form a complex with another Mst1 protein kinase [75]. This aspect is important due to the creation of conditions for dimerization, the key mechanism of interaction between MST kinases and RASSF proteins, which is crucial today. Dimerization is the basis for RASSF to participate in the regulation of the catalytic activity of MST kinases [84]. As it has already been established that RASSF proteins have several distinct domains and act as adapter proteins in many important biological processes, e.g. pro-apoptotic pathways, cell cycle and cytoskeleton regulation [85]. Interaction between some members of RASSF and various Ras proteins has been noted [85]. Ras association is likely to locates RASSF and MST kinases in the plasma membrane, bringing the MST kinase domains in close proximity to trigger phosphorylation transactivation, driving the MST/Hippo pathway and cellular apoptosis [50,71,84]. The binding time between Mst1 / 2 and RASSF allows the Hippo pathway to respond and integrate a variety of cellular signals [83].

\section{Core proteins of the hippo pathway as a therapeutic target in diabetes}

\subsection{The serine/threonine kinase, mammalian STE20-like kinase 1/2 (MST1/2 kinase)}

MST 1 and MST2 are the canonical core of Hippo signaling in mammals [89], and their operation has been repeatedly confirmed and is largely associated with organ size control [90-92]. Moreover they regulates: cell architecture, cell polarity and proliferation, homeostasis and cell death $[64,89]$. To date, five MST kinases have been described in mammals: MST1 (or STK4), MST2 (or STK3) of the germinal center kinase II (GCK II) and MST3 (or STK24), MST4 (or STK26), YSK1 (STK25/SOK1) representing the germinal center kinase III (GCK III) [93]. Both MST1 and MST2 are closely related class II GC kinases (Ser / Thr protein) [94]. In humans consist of 487 and 491 amino acids, respectively [95]. Between the $\mathrm{N}$-terminal and C-terminal regulatory domains are arranged: the auto-inhibition

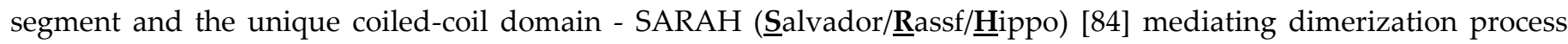
[93,94,96]. The SARAH domains ( 50 amino acids in length) mediate the homo- and heterodimerization of MST1/MST2 as they contain effectors RASSF binds to another protein kinase, MST1 [72]. Moreover, they are responsible for promoting protein-protein interactions between with other SARAH domain proteins [97]. It should be emphasized that MST1, as a core kinase of the Hippo pathway, is expressed in full length i.a. in the $\beta$ cells of the pancreas [59]. Scientific research contributed to the recognition of two caspase cleavage sites (D326 and D349) in the MST1/2 region between the kinase and the auto-inhibiting domain. Both internally arranged domains are removed in the process of caspase cleavage, and their elimination results in the activation of the core protein during apoptosis [98,99]. Thus, MST1 comes in two forms as: full length $54 \mathrm{kDa}$ protein and $36 \mathrm{kDa}$ caspase cleaved version [100]. MST1 isoforms show a different kinase activity (among themselves) in relation to substrates [101]. The caspase cleaved Mst1 fragment is not only more active than full-length because it lacks an auto-inhibition domain, but also has different 
substrate selectivity. Truncated Mst1 reduces the affinity for transcription factor forkhead box O (FOXO) while increasing the affinity for the nucleosomal component protein, histone H2B [64,97]. Removal of the above domains increases its activity approximately 9-fold compared to wild-type MST1 [96]. In addition, it was found to contribute to the creation of a positive feedback loop in the maintenance of the apoptotic signal through caspase activation as well as additional stimulation of MST1 cleavage [102]. The implementation of various apoptotic pathways was also observed, the course of which is associated with an increase in MST1 activity [59], therefore this kinase is considered a critical regulator of apoptotic death and $\beta$-cell dysfunction [102].

The adopted scheme of consequences related to the increased activity of MST1 in the diabetic environment (is induced by cellular stress of varius etiology, such as proinflammatory cytokines, glycolipotoxicity and oxidative stress) assumes the activation of mitochondrial-dependent apoptotic processes, mainly by targeting mitochondrial pro-apoptotic protein - Bcl-2-like protein 11 (BIM), which results in changes in Bax/Bcl-2, the release of cytochrome c, the cleavage of caspase 9 and -3, and consequently cell death [66]. Both human and mouse pancreatic islets as well as INS-1E cells exposed in vitro to diabetic conditions (complex conditions - more information [76]) have been shown to reveal a correlation between increased MST activation (in all cases) and $\beta$-cell apoptosis[76]. Ardestani et al. [76] indicate that it is an essential apoptotic molecule, induced in various diabetogenic states that are directly related to: impaired survival and Langerhans islet $\beta$ cell dysfunction in diabetes. Indicating that MST1 may be a common component of a variety of signaling pathways leading to apoptosis. In basic studies, it was shown that overexpression of MST1 in both human islets and INS-1E cells caused their apoptosis by caspase-3 cleavage and autophosporyaltion (pMST1-T183). MST1-induced apoptosis occurs via a mitochondrial-dependent pathway. The expression profile of internal death pathway regulators containing only BH3 showed only the highest induction of BIM. Pointing to the strong relationship in human pancreatic islets between MST1 and BIM which is regulated by c-jun N-terminal kinase (JNK) and AKT [76,103,104]. In antagonistic conditions - silencing (80\%) of MST1 in human pancreatic islets lowered the course of apoptosis, and a reduced value for BIM was also noted in diabetic conditions [76]. Caspase-3 and JNK initiate a vicious circle and pro-apoptotic signaling cascade in the $\beta$ cell by interacting with MST1 as both upstream activators and downstream targets. Cleavage of MST1 is induced by a variety of apoptotic signals, in vitro, including signals from the Fas receptor and TNF $\alpha$ [76,98]. It was confirmed that both Fas, caspase 3 and TNF $\alpha$ can induce apoptosis kinase via MST1 its role in this programmed process. MST1 and MST2 have been observed as direct substrates of caspase- 3 both in vivo and in vitro. has been shown to act as an up and down activator in the cell towards caspase activity, regulating its activation induced by the death receptor. Both kinases are proteolytically activated by specific cleavage and are involved in the Fas-mediated apoptosis process. The truncated form of MST or its overexpression leads to more dramatic morphological changes for $\beta$ cells and are similar to those caused by PAK2 (serine/threonine protein kinase) [98,105]. In conclusion, under resting conditions, MST1 is localized exclusively in the cytoplasm. Caspases mediate the cleavage of MST1, the effect of which is the removal of the C-terminal inhibitory domain and translocation of the active catalytic fragment into the nucleus $[59,98,100]$ and the change of localization may be associated with a different offer of substrates for phosphorylation [99].

On the other hand, ablation of MST1 revealed resistance to induced apoptosis by: TNF $\alpha$, Fas and IFN $\gamma$ [106-108]. In mice model of the deletion of Mst1 (knockout mice MST1 -/-) with diabetes induced experimentally (MLD-STZ) were not observed the loss of $\beta$ cells. Analysis of the architecture of the pancreatic islets of these animals revealed preserved structure, density, size and mass. It is also interesting that the $\beta$ cells functionally retained their function. In summary, genetically induced MST1 deficiency normalizes glycemia and restores the function of $\beta$ cells and prevents the development of diabetes [76].

MST1 regulates the activity of factors that enable the proper functioning and survival of $\beta$ cells. It has been shown to have a negative effect on PDX1 (duodenal pancreatic homeobox-1), a key transcription factor important for 
the development and functioning of $\beta$ cells. The high activity of MST1 contributes to the phosphorylation of PDX1 (at the T11 site), leading to its degradation by the proteasome machinery, preventing it from acting as a transcription factor in the nucleus. PDX1 degradation is accomplished via the ubiquitin-proteasome pathway. Unfortunately, the ultimate consequences are manifested by disorders of insulin secretion and the development of diabetes mellitus. Knockdown MST1 in mice normalizes PDX1 under diabetic conditions [76]. One of the mechanisms of protecting $\beta$ cells from MST1 depletion is the preservation of PDX1. In vivo and in vitro analyzes in Mst1 -/- mice showed normal blood glucose levels and increased circulating insulin levels. In addition, it has been shown that PDX1 target genes have been normalized and the location of GLUT2 is conserved. On the other hand, it influences the implementation of the phosphatidylinositol-3 kinase PI3K/AKT pathway, which is significantly related to the survival of $\beta$ cells. The serine-threonine kinases (AKT) regulate various cellular events, including metabolism by phosphorylation of a number of other substrates, their key functions indirectly influence the efficiency of functions and the survival of secretory cells. The cleaved form of MST1, an active form acts as an AKT inhibitor. Whereas, AKT-mediated phosphorylation negatively regulates MST1 activity and impairs nuclear translocation $[76,109]$.

\subsubsection{Therapeutic methods targeting MST1}

The empirical knowledge base accumulated in the basic research characterizing the properties of MST1 is a valuable starting point and the basis for designing therapeutic strategies dependent on its inhibition. In the last few years, in vitro and in vivo studies conducted on various research materials, including pancreatic islets obtained from animals and patients with type 2 diabetes, have shown that pre-diabetes conditions and those that reflect full-blown diabetes activate MST1. The presence of chronic diabetic lesions strongly increases the autophosphorylation of MST1 (T183) and induces programmed cell death [110]. It is worth recalling here that overexpression of MST1 induces apoptosis in a manner independent of diabetogenic conditions [76]. The above observations directly indicate that the major core kinase of the Hippo pathway is a versatile molecular tool and a valuable therapeutic target [111]. The observed effects related to its activity in diabetic states disrupt the secretion and action of insulin, and consequently many systemic changes lead to $\beta$ cell apoptosis [76]. In fact, as a central mediator of the Hippo pathway, it influences various mechanisms of apoptosis. The therapeutic benefits and hopes are not only directed at the $\beta$ cells of the pancreas in diabetes, but may also apply to diabetes complications including nephropathy and cardiomyopathy [59]. Loss of kinase (MST1-knockout (MST1-KO) mice) prevents the progression of diabetes and restores normal cell function [76]. A full description of its functions will facilitate the selection of factors with which to design and implement breakthrough therapies for various diseases, including diabetes. The starting point for the formulation of assumptions regarding the modulation of Hippo pathway signals in diabetes is the use of factors with a protective effect on pancreatic $\beta$ cells. The basis of this conclusion are the results of genetic MST1 deficiency, which brought beneficial effects in the form of glycemic normalization and improvement of $\beta$-cell function, survival and mass, and the inhibition of diabetes development [76]. The development of drugs to target MST1 kinase is still in its infancy and so far no drugs are available admitted to trading. The search for inhibitors to function as the specific drugs in the future involves essentially two extremely different approaches. The first is the synthesis of new selective ones molecules dedicated to the pharmacotherapy of diabetes, while the second strategy is based on the search for alternative indications for previously used medicinal substances in the pharmacotherapy of other diseases. The advantage of the second strategy is the well-known safety profile of the drug, which consequently significantly reduces the time needed to develop a new drug form. Several small molecule MST inhibitors have been described and their activity profile is established in the preclinical phase. [112,113]. The common denominator of MST1 inhibitors (presented in this review) is the documented ability to reduce its activity in the pancreas. The presented group of small molecules is 
not pharmacologically related, and their action has been presented at various stages of research advancement. Therefore, the form of their presentation maintains an independent character, unusual for discussion.

Among them, the most advanced neratinib, which is an inhibitor of tyrosine kinases anti-cancer medication [110,111]. Multitarget tyrosine kinase inhibitors (TKIs), as the second group of small molecules (with a wide range of factors available), have been reported for years as potential candidates in the treatment of diabetes symptoms [114,115]. Clinical trials have shown a beneficial antidiabetic effect in patients with type 2 diabetes who had their symptoms relieved from imatinib, sunitinib, desatinib and elrotinib [116-120]. Although today the main goal is cancer therapy, and their action is based on ERBB2/EGFR inhibition, it has been noticed (in clinical observations) that they additionally alleviate the symptoms of diabetes [121,122]. Increased EGFR contributes to insulin resistance and correlates with a higher risk of developing diabetes, and the use of TKIs inhibitors in most cases led to an arrest of diabetes development, normalization of glycemia, and in extreme cases even diabetes re-emission. Some of the TKIs have already been thoroughly verified and approved by the FDA, which is an added bonus [115,122]. Preclinical studies of these factors have shown an inhibitory effect on apoptosis of pancreatic $\beta$ cells in animals and humans, thus eliminating one of the main causes of diabetes [123]. Intensive verification of the effects associated with the presence of neratinib showed a strong inhibition of MST1, which resulted in improved survival of $\beta$ cells in vitro and became the basis for the observation of molecular sequelae [110]. In contrast, in vivo analyzes showed an improvement in insulin resistance by inhibiting the production of tumor necrosis factor alpha (TNF- $\alpha$ ) or by reducing the stress of the endoplasmic reticulum [124-126]. Its main use is in blocking the growth of the human epidermal factor 2 receptor (ERBB2, HER2) and the epidermal growth factor receptor in the treatment of breast cancer [127-129] as a drug approved by the Food and Drug Administration (February 25, 2020 as NERLYNX, Puma Biotechnology, Inc.) and has achieved the Tracking State rate. Demonstrated clinical trials (https://clinicaltrials.gov, as of 23-10-2021) include 69 trials at various stages of implementation and advancement (early phase 1-phase 3). Although clinical trials targeting cancer have shown a potential anti-diabetic effect, typical analyzes focusing solely on diabetes parameters have yet to be initiated. Nevertheless, it seems that such studies are only a matter of the near future.

Basic research focused on the use of neratinib as an MST1 inhibitor in the Hippo pathway under various experimental conditions, including diabetic conditions, shows promising prospects and relief of underlying symptoms. No toxic effects were observed during the performance of screening analyzes to select the effective concentration for studies with isolated pancreatic islets (25 $\mu \mathrm{M}$ neratinib) and for intraperitoneal injections (marix-assisted laser desorption ionization imaging mass spectrometry (MALDI-IMS)). The specificity of the action on human and murine pancreatic $\beta$ cells revealed an anti-apoptotic effect and increased survival reflecting the action of neratinib in diabetogenic states. While no side effects were observed in the control groups, including a reduction in glucose levels or $\beta$ cell function. Moreover, under high glucose conditions, neratinib restored PDX1 expression and increased cell viability. Caspase-3 activity reflecting ER stress (experimentally induced) was also suppressed by neratinib in a dose-dependent manner, highlighting the many benefits of inhibiting pre- and diabetic activated MST1. In addition, neratinib was effective in counteracting stress-induced MST1 activation, and apoptotic effectors cleaved caspase-3 and PARP. Similar observations, i.e. inhibition of proinflammatory cytokine activation, MST1, and caspase-3 activation, were demonstrated in human pancreatic islets incubated with neratinib under high glucose/palmitate conditions. Comparable observations have been made in studies conducted in experimental MST1 knockout mouse models. Despite strong activating factors of apoptotic pathways (including pro-inflammatory cytokines and glycolipotoxicity), pancreatic islets from MST1-KO mice did not reveal apoptosis, unlike in WT. Under diabetogenic conditions, neratinib reduced apoptosis but had no significant effect in MST1-KO mice. The implementation of apoptosis, under conditions of MST1 overexpression, was blocked by neratinib in in vitro experiments performed on $\beta$-cells of isolated human islets. Blocking the apoptotic pathway or alleviating the 
symptoms and causes of diabetes are groundbreaking and fundamental goals for the treatment of diabetes. For neratinib, many have already been reported. Nevertheless, it is important that the effects achieved at the laboratory level turn out to be permanent and fully recognized at the molecular level. Therefore, analyzes were also performed to identify molecular triggered by neratinib. Real-time analysis of INS-1E cells with adenoviral overexpression of MST1/LATS2 transfected with LATS-BS revealed specific apoptotic consequences dependent on these two essential proteins of the HIPPO pathway. Revealing MST1-LATS2 interoperability activating downstream processes. Only neratinib (compared to the lack of action of the EGFR inhibitor canertinib) in this experimental system strongly inhibited both kinases, thus blocking the continuation of this process. In experiments carried out mice with experimentally induced MLD-STZ type 1 and type 2 diabetes, Neratinib improved glycemia, insulin secretion, and $\beta$-cell survival in a mouse model of MLD-STZ type 1 diabetes. In models reflecting the conditions of type 2 diabetes, the pancreatic islet architecture of mild-dose MLD-STZ diabetes mice is characterized by structural abnormalities, including a reduction in both the number of $\beta$ cells and the expression of important markers of glucose metabolism in cells that have retained secretory function (PDX), NKX6.1 and the glucose transporter GLUT2). Neratinib restored cell function and increased cell survival. Additionally, it restored the virtually lost expression of NKX6.1, as well as PDX1 and GLUT2. In another experiment, Ardestani et al. [110] showed that neratinib (5 mg/kg administered intraperitoneally) in the treatment of diabetes in the obese diabetic Leprdb/db mice model counteracted high hyperglycemia in these animals. Neratinib was administered to obese $\mathrm{db} / \mathrm{db}$ mice (animals 6 weeks old) for a period of 31 days. Stability in blood glucose levels was observed in relation to the vehicle control group and no change in body weight. In addition, a group of animals treated in the intraperitoneal glucose tolerance test showed an amelioration of hyperglycemia as well as increased insulin secretion at all measured time points. In an intraperitoneal insulin tolerance test, it was revealed that mice treated with neratinib showed a slightly reduced ability to lower glucose in response to insulin. Analysis of changes in $\beta$ cells after neratinib therapy allowed to observe increased $\beta$-cell mass as an effect reflecting decreased $\beta$ cell apoptosis, as well as increased proliferation (Ki67 markers used and phospho-histone H3 (pHH3) immunolabeling). The presence of PDX1 has also been noted in the nucleus, which has been boosted by neratinib. Ex vivo experiments carried out on isolated pancreatic islets revealed a normalizing effect in severe states of diabetes. Langerhans' islets of advanced diabetic $\mathrm{db} / \mathrm{db}$ mice with an increased level of $\beta$-cell apoptosis (3.2-fold increased) compared to the control group ( $\mathrm{db} /+$ mice) were treated with neratinib. Its presence inhibited the activation of MST1 and the pro-apoptotic BIM protein and contributed to the full restoration of $\beta$-cell survival. Additionally, an increase in nuclear PDX1 expression was observed. Another experiment examined whether cytokine-induced $\beta$ cell apoptosis could be reversed by the action of neratinib. Pancreatic islets treated with the IL-1 $\beta /$ IFN $\gamma$ mixture of cytokines revealed a sharp increase in apoptosis, however restoration of $\beta$ cell survival was observed in some of the neratinib experimental groups [110].

The second factor that also presents promising prospects is XMU-MP-1 [130,131]. Previous studies have demonstrated the pharmacokinetic efficacy of XMU-MP-1 in inhibiting MST1/2 in mice, further revealing liver and intestinal regeneration in vivo. Pharmacological manipulation of Hippo's signaling pathway with XMU-MP-1 offers a broad spectrum and opens up new possibilities for research in regenerative medicine, apoptosis, and certainly more. Especially that the proposed inhibitor seems to be a safe targeted therapeutic agent in the treatment of tissue injuries. The action of XMU-MP-1 mainly targets MST1/2 and inhibits both kinases with IC50 values of $71 \mathrm{nM}$ and $38 \mathrm{nM}$, respectively, although inhibition of MOB1 protein phosphorylation was observed in a dose dependent manner [131]. Incubation of INS-1 cells with increasing doses of XMU-MP-1 (1-5 $\mu \mathrm{M})$ showed MST1/MST2 inhibition and related sequelae. Reduced phosphorylation of other MST1/MST2 substrates, ie LATS1 and MOB1, has been demonstrated. In addition, a dose-dependent increase in YAP activity was observed [130,131]. The MTT test revealed that lower doses of XMU-MP-1 are not toxic to INS-1 cells, although its concentrations of 3-5 $\mu \mathrm{M}$ decreased cell viability, and the 
highest dose of $5 \mu \mathrm{M}$ caused the loss of 30\% of INS-1 cells (24h incubation). XMU-MP-1 at various concentrations of 1-3 $\mu \mathrm{M}$ increased the survival of INS-1 cells previously treated with STZ (1-2 mM for 24 hours). In vivo experiments were carried out in wild-type Balb/c mice with experimentally induced STZ diabetes (at a dose of $50 \mathrm{mg} / \mathrm{kg}$ body weight). In subjects with fully developed diabetes symptoms, XMU-MP-1 (at a dose of $1 \mathrm{mg} / \mathrm{kg}$ bw/day) or DMSO (control group) was administered for 21 consecutive days. Fasting blood glucose analysis as well as glucose tolerance test (GTT) or bodyweight monitoring, in the initial phase of the experiment, showed no differences in blood glucose levels between the XMU-MP-1 group and the DMSO group. Reassessment (after 35 days) of fasting glucose and the glucose tolerance test showed positive effects of XMU in both groups of experimentally induced diabetes animals (mild and severe form) compared to the control group (DMSO). Noteworthy is also the speed of regulation of blood glucose levels (15 minutes from intraperitoneal administration) to intraperitoneal glucose. Blood glucose analysis in animals treated with XMU stabilized in a much shorter time than in the control group. Comparison of the effects between animals showing mild diabetes versus those with acute diabetes mellitus GTT results revealed that the beneficial effects of XMU-MP-1 were only observed in the severe diabetic group. Analysis of histological sections of Langerhans pancreatic islets from mice treated with XMU-MP-1 showed no statistically significant differences in terms of the mean number of islet cells and pancreatic islet surfaces in non-diabetic and experimentally induced diabetes mice. Detailed analysis of preparations from animals with acute diabetes mellitus showed the beneficial effect of XMU-MP-1, which was revealed as an increased cell number and islet area. In vivo studies of the effectiveness of the XMU-MP-1 inhibitor showed its greater effectiveness only in the group with severe diabetes [130].

The final inhibitor for MST1 is Asialo-erythropoietin [132], an EPO derivative with no sialic acid residue. It does not exhibit hematopoietic properties and is characterized by a very short circulating half-life [133]. The proposed molecule is a cytokine with a broad neuroprotective effect [134]. Among the group of derivatives of its group, it shows the best protective effect against diabetes-induced apoptosis in Rin-m5F cells, therefore its action is presented in this review. In in vitro experiments, RIN-m5F cultured cells were incubated with both asialorhuEPOP at various concentrations $(20,40,60,80$ or $100 \mathrm{IU} / \mathrm{ml}$ ) and STS (staurosporine) for 24 hours. The changes in cytotoxicity in the control group cells - treated with STS alone amounted to 46\%, while the simultaneous exposure of STS and 20-100 IU asialorhuEPOP caused reduced cytotoxicity (in the range of 40-20\%). While the cytoprotection index revealed the highest values for the doses of 60,80 or $100 \mathrm{IU} / \mathrm{ml}$ asialorhuEPOP and was $41-56 \%$. The protective effect of asialo-rhuEPOP is due to its ability to inhibit MST1 and caspase -3 under diabetic conditions, which was confirmed by a significant reduction in the amount of cleaved MST1 and cleaved caspase-3 compared to control cells (incubation only with STS). Asialor-rhuEPOP under the conditions of induced diabetes reduces the expression of the pro-apoptotic protein Bax and increases the amount of the anti-apoptotic Bcl-2, which was demonstrated in the analysis of the expression profile of mitochondrial proteins. Additionally, its anti-apoptotic effect is related to the preservation of AKT phosphorylation. Moreover, it increased the amount of PDX-1 protecting it from degradation, and the obtained value is comparable to the control levels. In contrast, the decreased insulin secretion (30\%) due to STS was restored by asialorhuEPOP to approximately $22 \%$ in Rin-m5F cells [132].

\subsection{Large tumor supresor $1 / 2$ (LATS 1/2)}

The large tumor suppressor (LATS) gene family, including LATS1 and LATS2, an MST1 downstream substrate, is an essential component of the Hippo pathway [135]. These proteins are critical regulators of cell apoptosis, originally identified in fruit flies [136]. It belongs to the serine-threonine protein kinases. Human LATS1 and LATS2 belong to the AGC subfamily, related to Dbf2-related nuclear kinases (NDR1/2) [137]. Both kinases show significant 
homology ( $85 \%$ similarity) in the sequence of the kinase domain located at the C-terminus of the proteins. In contrast, the N-terminal shows much less maintenance [138]. Their structure includes: C-terminal kinase domain, protein binding domain (PBD), two conserved LATS domains (LCD1 and LCD2), ubiquitin binding domain (UBA) and at least one PPXY motif (P: proline, X: any amino acid, Y: tyrosine), which binds to the YAP and TAZ domains. The C domain has two conserved Ser/Thr phosphorylation sites. The site of autophosphorylation is serine, while threonine is the site of phosphorylation by MST1 and MST2. At the amino terminus (N), there are two stretches of conserved sequences (LCD1 and 2), and their presence is essential for the proper regulation and function of the core kinases of the HIPPO pathway [139]. Moreover, both LATS1/2 within the $N$ terminus exhibit evolutionarily conserved ubiquitin-related domains. The presence of such domains enables the binding of the ubiquitinated protein and may therefore be involved in their activation [137,140]. Both isoforms of kinases have unique features in their structure, which are the basis of various protein-protein interactions. At its N-terminus, LATS1 has a proline-rich domain, while LATS2 contains the unique PAPA repeat. Additionally, both kinases were observed to code for the PPxY motif that determines interaction with downstream components of the HIPPO pathway, including YAP and TAZ [141]. The hydrophobic motif of both kinases is similar to other AGC kinases such as AKT, S6K1 (important fo mTORC1) and PKC [64]. LATS1 and LATS2 constitute a unique family of signaling in the cell, they are important signaling molecules, and their action is aimed at the regulation of transcription and maintenance of genetic stability, modulation of cell cycle checkpoints, induction of apoptosis [142-144]. Moreover, these proteins are involved in the regulation of: cell proliferation and apoptosis, therefore a conservative belief prevails, which is not entirely correct, that they are mainly involved in the development of cancer [142]. The tremendous metabolic plasticity of pancreatic $\beta$ cells and their response to stress, followed by pro-diabetic signals, is driven by the structure and spacetime dynamics of complex signal transduction networks. These factors influence the components of the HIPPO pathway by modulating its. Research has shown that NF2 is an upstream regulator in Hippo's signaling pathway [145]. It was found to be expressed in cellular models - INS-1E, as well as in primary human islets. Loss of NF2 function in pancreatic cells can overcome cell apoptosis and is revealed by inhibition of LATS2 activity. This molecular modulation has no effect on cell function as well as on the functional genes of the cell identity [87]. LATS-regulated cell apoptosis also affects downstream targets, the most common of which is P53, FOXO1, abelson tyrosine kinase c-Abl (c-Abl) and YAP [146-148]. LATS is a critical component of the HIPPO pathway for the induction of apoptosis in $\beta$ cells. Its mechanism of action is based on mechanistic target of the rapamycin 1 (mTORC1). The central kinase responsible for regulating cell growth in mammals is the mechanistic target of rapamycin, also called the mammalian target of rapamycin (mTOR). So far, two different complexes have been described: mTORC1 and mTORC2, and the difference between them is due to the presence of a unique helper protein, Raptor and Rictor, respectively [149]. mTORC1 is responsible mainly for the regulation of cell growth and metabolism, and mTORC2 for the control of proliferation, and is also responsible for cell survival [150]. The mTOR pathway is activated by energy input, especially in two important forms: amino acids and insulin-like growth factors (IGFs) [149]. It is believed as mediating $\beta$ cell growth and expansion [149]. However, its excessive activation and negative effects related to the loss of $\beta$ cells were revealed in animal models showing the presence of type 2 diabetes [79]. Clearly pointing to an ongoing apoptosis scenario in which the presence of conditions leading to diabetes activates the sequelae cascade, i.e. diabetic conditions activate LATS and thereby activate mTORC1 by suppressing AMP-activated protein kinase (AMPK) signaling. Moreover, growing evidence suggests that AMP-activated protein kinase (AMPK) and mTOR play a pivotal role in autophagy and apoptosis [151].

\subsubsection{Therapeutic methods targeting LATS}


Research focused on the role of LATS1/2 in the process of apotosis of pancreatic $\beta$ cells is still carried out in the convention of understanding the mechanism of regulation of this kinase in this process. Despite the fact that the first inhibitors are already used in other scientific fields (POSZUKAJ CYTOWANIA INHIBITORY LATS). Yuan et al. [79] showed that large tumor suppressor 2 (LATS2) induces apoptosis, but also affects pancreatic $\beta$ cells by participating in the development of apoptosis. The action of this isoform is revealed in diabetic states. INS-1E cells and cells derived from human pancreatic islets have been shown to be highly susceptible to LATS2 deficiency, resulting in improved viability, increased cell mass, and restoration of insulin secretion, combined to reduce the development of diabetes. LATS1/2 kinase activity results in phosphorylation of YAP targets on S127 (LATS specific site) resulting in the exposed docking site of the 14-3-3 proteins. As a result, YAP cytoplasmic sequestration occurs. LATS kinase inhibitors used so far have found application in research on: neoplastic changes and proliferation.

Few inhibitors targeting the LATS kinase have been designed so far, which is directly related to the lack of a fully known crystal structure of this kinase [152] and constitutes a serious obstacle to the formulation of a specific action molecule. More recently, information has appeared in the literature about a TRULI inhibitor obtained from Enamine LLC (Z730688380, Monmouth Junction, NJ) and targeting LATS1 and LATS2 [152]. Relatively recently (WO / 2018/198077), 6-6 fused bicyclic heteroaryl compounds have been patented, the recommended therapeutic applications of which are related to the healing of various wounds $[153,154]$.

There are tons of articles in digital databases describing the function of LATS1/2 in pancreatic proliferation or cancer, which unfortunately does not apply to diabetes. To our knowledge, supported by a detailed analysis of the many available databases containing articles in the field of medicine and life sciences, so far only one pioneering publication [79] detailing the operation of LATS has appeared. Extensive studies have been performed on $\beta$ cells, isolated human and murine pancreatic islets, and diabetic mice or other genetically generated model. The interpretation of LATS2 action in diabetes and apoptosis has been confronted with an explanation of the role and interaction of mTOR and autophages in these processes. Despite the fact that there are many documented experiences regarding the function of the mTOR pathway itself and autophages in this specific area, it is difficult to refer to them as they do not directly discuss the function of the HIPPO pathway, although it may be assumed that the effects obtained are due to its involvement.

LATS2 as one of the core kinases of the HIPPO pathway is responsible for interfering with the survival and functioning of $\beta$ cells. The above finding is confirmed by both in vitro and in vivo experiments, which have shown that diabetogenic states increase the activation of LATS. The effects of glucotoxicity and glycolipotoxicity were the direct cause of the induction of apoptosis and were also associated with loss of $\beta$ cell function due to LATS1/2 overactivity. The increase in kinase activity produced a "ripple effect", ie LATS dependent: increased YAP phosphorylation and increased MOB1 levels. Other experiments on INS-1E and human pancreatic islets revealed an increase in MOB1 levels as a result of LATS2 overexpression alone (induced by adenoviral transduction). Overexpression of LATS causes apoptosis, as evidenced by the split caspase-3 and poly (ADP-ribose) polymerases (PARP), another caspase-3 substrate, present in the cell. Also in the isoaleted pancreatic islets of obese Lepr ${ }^{\mathrm{db} / \mathrm{db}}(\mathrm{db} / \mathrm{db})$ mice, it was confirmed that the level of exogenously expressed pYAP and MOB1 proteins was increased. This finding was confirmed by experiments in which the loss of endogenous LATS2 activity protected the tested INS-1E cells against apoptosis, despite the diabetogenic conditions of their culture. The conclusion was supported by the results of the experiment in which the loss of endogenous LATS2 activity shielded and moderated the development of apoptosis in INS-1E cells, even indicating a pro-survival effect, despite the unfavorable diabetogenic conditions of their culture. In addition, $\beta$ cells have shown a potential difference in the regulation of apoptosis between LATS1 and LATS2 [79]. 
Multiple pathways can be crucial for the induction and execution of the very complex process of apoptosis. Collective action is often filled with dependencies in the course of molecular mechanisms, creating the basis for mutual control. As research in this field in the area of $\beta$ cells is still clarifying, and on the other hand, it is impossible to mention all possible interactions, so below will be those whose operation is critical to revealing or modifying the LATS function. An example of such an interaction in the $\beta$ pancreas is the mTORC1 - HIPPO LATS crosstalk. Analyzes conducted by Yuan et al. [79] found that LATS2 acts as a key upstream activator for the mTORC1 pathway in $\beta$ cells exposed to stress and the knockout of HIPPO kinase was significant for weakening downstream signaling, among others mTORC1. The arrest of the mTORC1 pathway was revealed by the blocking of $\beta$ cell apoptosis, which clearly showed that the pro-apoptotic effect of LATS2 is mTORC1 dependent and is over-activated by LATS2 in diabetes in INS-1E cells and human pancreatic islets [13]. The development of apoptosis in $\beta$ cells and isolated human pancreatic islets grown under proapotoxic (glucotoxic) conditions revealed the implementation of mTORC1 in a LATS2 dependent manner. Silencing of mTORC1 resulted in an arrest of apoptosis despite pancreatic $\beta$ cell toxic conditions and LATS activation. On the other hand, it was revealed that overexpression of LATS2 in these cells activated mTORC1. The action of LATS in diabetic conditions leads to apoptosis mediated by activation of Rag-mTORC1 [79]

Autophagy is responsible for the functionality of cell organelles essential for the survival/functioning of $\beta$ cells. Their function is also important in improving insulin sensitivity during a high-fat diet in mice [155] but in diabetes, however, it plays a role in its pathogenesis [156]. It has been shown that autophagy can protect diabetes in a manner independent of mTOR. On the other hand, both insulin and mTOR, are inhibitors of autophagy. The haploinsufficiency of autophagy in murine animal models of obesity increased insulin resistance, which was accompanied by elevated lipid levels, as well as inflammation [156]. It has been observed that the elimination of both misfolded proteins and mitochondria to protect $\beta$ cells from loss of function in diabetes is largely done by autophages $[157,158]$. Thus, loss of autophagy is what causes obesity-induced diabetes to develop. Ardestani et al. [159] demonstrate a high functional relationship between mTOR and autophagy. The mechanism induced by insufficiently elevated levels of nutrients leading to long-term activation of mTORC1 likely leads to $\beta$ cell failure, which is the opposite of how this pathway works normally. The prolonged state of increased mTORC1 activity is manifested by a progressive loss of: function, $\beta$ cell mass and associated mTORC2 impairment and mTOR-mediated autophages [159]. Studies focused on the role of LATS2 as an activator of mTORC1 revealed an impairment of autophagy in $\beta$ cells, which led to reduced viability of these cells and at the same time revealed another potential mechanism of counteracting. In vitro experiments revealed apoptosis in $\beta$ INS-1E cells and isolated human islets by simultaneous inhibiting autophagy and overexpression of LATS2. Under the conditions of LATS2 silence and autophagy inhibition, the course of apoptosis was reduced. As apoptosis increased, LATS2 decreased autophagic flow in $\beta$ cells and human pancreatic islets. Accumulation of autophagic flux markers (1A/1B-light chain 3 (LC3-II) and p62 (SQSTM1)) under LATS2 overexpression is also evidence of attenuated autophagic flux dependent on increased HIPPO core kinase activity. Moreover, p62 is an integral part of the mTORC1 complex and it interacts in an amino acid-dependent manner with mTOR and the raptor, confirming the mediation of this pathway between LATS2 and autophagy. Conversely, the loss of LATS2 in human islets decreased the accumulation of LC3-BII and p62 proteins caused by autophagy inhibitors. The above data prove a significant role of LATS2 in autophagy flow and at the same time indicate a participation in the implementation of apoptosis induced by defective autophagy. It is also interesting that LATS2 is located in lysosomes and acts as a substrate for autophagy in the degradation process. Moreover, it was confirmed that in the pancreas under the conditions of increased LATS2 in the pancreatic $\beta$ cells, macautophage realization dominates as one of the two mechanisms of autophage [79]. 
In vivo experiments were performed in a mouse model with a $\beta$ cell-specific LATS2 deletion ( $\beta$-LATS2 -/-) and the LATS2fl/fl control group with MLD-STZ experimentally induced diabetes. While control animals showed progressive development of diabetes (hyperglycemia, severely impaired glucose tolerance, impaired insulin secretion), $\beta$ cell LATS2 deficient mice showed milder changes in diabetes development induced by low doses of STZ. Analysis of $\beta$ cell mass quantification on histological slides in $\beta$-LATS2 -/- and LATS2 fl/fl mice showed a significantly reduced $\beta$-cell mass in control mice. Moreover, further analyzes showed that the pancreatic $\beta$ cell mass of LATS2 ablated mice were a result of increased proliferation and reduced apoptosis. The above results clearly indicate that the deficiency of LATS in the pancreas has a protective effect on $\beta$ cells in diabetes [79]. Similar results were obtained in animals fed 17 weeks of a high fat/high sucrose diet, which in control animals contributed to the development of insulin resistance, $\beta$ cell failure and hyperglycaemia. The development of symptoms was accompanied by diet-induced LATS2 activity. Animals with the LATS -/- genotype under the same experimental conditions showed a significant improved glucose tolerance, higher insulin secretion, and a positive proliferation/apoptosis ratio compared to control animals. Moreover, immunohistochemical analyzes of the $\beta$ islet cells of high fat/high sucrose fed mice showed a significantly higher expression of the phosphorylated form ribosomal protein S6 (pS6) which is a reliable marker of mTORC1 and S6K activation compared to the group of control animals. Different results were observed in HFD-fed $\beta$-LATS2 -/- mice in which pS6 expression was normalized. LATS2 deficiency blocks mTORC1 implementation in high fat/sucrose fed mice, furthermore maintains the interaction between LATS2 and the mTORC1 autophagy axis [79].

All above studies provide the basis for the conclusion that both the HIPPO-LATS and mTORC1 pathway kinase as well as autophagy co-create signaling related to pancreatic b cell survival susceptible to stress factors. The protective effect of autophagy is based on the positive feedback directed to LATS2 and the cell cover under conditions of severe stress. Nevertheless, such a mechanism of action does not function in long-term stress (e.g. diabetes), the effect of which is the development of apoptosis through the activation of LATS2, mTORC1, which results in the implementation of defective autophagy. In conclusion, LATS2 may be a second therapeutic target alongside MST1 whose blockage of action may lead to improved function and increase beta-cell survival in diabetes [79].

\subsection{Yes-associated protein (YAP)}

Two YAP isoforms (YAP1 and YAP2) have been described so far [160]. They show some structural differences related to the WW domain, the presence of one described for YAP1 and two for YAP2. In both the N to C terminal isoforms a proline-rich region, TEAD binding domain, SH3 binding motif, transcription activation domain, PDZ binding motif have been recognized [161]. In addition, YAP as a transcriptional co-activator has a transcription activation domain but no DNA binding domain [162]. The YAP protein is considered to be an analog of TAZ and their functions are referred to as redundant and usually formulated as YAP/TAZ [163,164]. Although they may function independently, YAP is considered to have a stronger effect than TAZ. Similarities in topological structure and the presence of about half of the identical oxygen sequences were recognized between YAP1 and TAZ [164]. The major regulator of YAP/TAZ through phosphorylation is the kinase cascade of the HIPPO pathway [165]. Phosphorylation of YAP and TAZ (=inactivation) by LATS1/2 (Yap on S127 or TAZ on S89) results in binding of the 14-3-3 protein, and sequestration of YAP in the cytoplasm results in ubiquitin-dependent degradation [90]. When the Hippo pathway kinases are inactive, the downstream effectors YAP and TAZ are located in the nucleus and activate transcription programs in cooperation with the transcription factors of the TEA domain (canonical pathway) [166]. Literature data indicate that there are more transcription factors apart from the TEAD family mentioned above, including P73 and Runx2 cells causing proliferation and survival [92]. The participation of AMPK was also found, the effect of which in this area is inhibition of cell growth, and due to YAP phosphorylation it leads to the disruption of YAP-TEAD [167]. 
The scope of its action is very wide and is mainly related to driving embryonic growth [168,169], stem cell proliferation and differentiation [170,171], vascular remodeling [172], and its role in the development of diseases of the nervous system and cancer has been described [173]. Moreover YAP/TAZ stand out as elements of the HIPPO cascade involved in the regulation of organ location and size, as well as tissue regeneration [174,175]. Thus, YAP/TAZ dysfunction is causing an increasing number of human diseases [176]. Although its basic action is still understood stereotypically and associated mainly with embryogenesis or cancer, in the last few years there will be proposals to use its potential in the treatment of patients with diabetes, hypertension and metabolic syndrome in the prevention of heart failure syndromes [177]. Manipulating the activity of YAP, e.g. which is considered by many to be an oncoprotein, is not safe without taking appropriate precautions, including targeted action or activity control. The YAP function changes drastically depending on the type of stress [177], and also shows significant sensitivity to high/low glucose levels in insuli target cells [178], , therefore its mechanism of action should be carefully investigated. It was also observed that it was activated in patients with heart failure and diabetes [177]. Other studies have shown that YAP plays an important role in both the replication and survival of $\beta$ cells. YAP-induced $\beta$-cell proliferation has been diagnosed in adult studies and has been involved in the protective functions against the pro-apoptotic effects activated by diabetes [179]. All indications are that YAP activity is perceived as timely and activated under conditions of demand, e.g. regeneration of $\beta$ cells damaged in diabetic conditions. The relationship with many physiological processes, including growth stimulation, regulation of the cell cycle, or proliferation, is a kind of functional showcase of YAP and evidence of interaction with many signaling networks [179-184]. The forkhead box M1 protein (FOXM1) was also noted in the YAP-dependent process of pancreatic beta cells proliferation. FOXM1, as a member of the FOX family, plays an important role in the progression of the cell cycle, and its significant effect has also been noticed in postnatal proliferation and mass expansion of $\beta$ cells exposed to increased stress in vivo [179,185]. Oxidative stress as one of the many factors leading to the deterioration of $\beta$ cell function during the development of type 2 diabetes can be prevented by the action of thioredoxin (Trx), a redox protein expressed in pancreatic $\beta$ cells. Most likely, the operation of Trx1 is YAP dependent, and the participation of them in specific regulatory signal loops complex. In vivo studies in rodent models with type 1 and type 2 diabetes have shown that $\beta$-cell-specific Trx 1 overexpression inhibits their death and slow the progression for type 1 diabetes [186,187]. In contrast, silencing Trx1/2 lowered exogenously expressed YAP. On the other hand, induction of YAP overexpression in healthy and diabetic human islets and cultured $\beta$ INS-1E cells resulted in an increase in Trx1/2 activity.

As demonstrated above, the HIPPO pathway is a huge potential and future in the treatment of type 2 diabetes. Using its possibilities will certainly break the usual patterns of diabetes treatment. Modulations in its activity at different levels of core kinases may: inhibit apoptosis, and thus the loss of insulin-secreting cells, while restoring their full functionality.

Author Contributions: All the necessary stages of creating this publication were carried out in cooperation with A.K. and A.Z. The final version of the article was approved by both authors. All authors have read and agreed to the published version of the manuscript.

Conflicts of Interest: The authors declare no conflict of interest

Appendix B Figure 1

\section{References}

1. Yang, H.; Yang, L. Targeting cAMP/PKA pathway for glycemic control and type 2 diabetes therapy. $J$. Mol. Endocrinol. 2016, 57, R93-R108. 
2. Wang, C. C. L.; Hess, C. N.; Hiatt, W. R.; Goldfine, A. B. Clinical Update: Cardiovascular Disease in Diabetes Mellitus. Circulation 2016, 133, 2459-2502.

3. Kilanowska, A.; Szkudelski, T. Effects of inhibition of phosphodiesterase 3B in pancreatic islets on insulin secretion: a potential link with some stimulatory pathways. Arch. Physiol. Biochem. 2019.

4. Kilanowska, A.; Ziółkowska, A. Role of Phosphodiesterase in the Biology and Pathology of Diabetes. Int. J. Mol. Sci. 2020, 21, 2-26.

5. Feng, X.; Wang, S.; Yang, X.; Lin, J.; Man, W.; Dong, Y. Mst1 Knockout Alleviates Mitochondrial Fission and Mitigates Left Ventricular Remodeling in the Development of Diabetic Cardiomyopathy. Front. Cell Dev. Biol. 2021, 8, 628842.

6. Sifuentes-Franco, S.; Padilla-Tejeda, D. E.; Carrillo-Ibarra, S.; Miranda-Díaz, A. G. Review Article Oxidative Stress , Apoptosis , and Mitochondrial Function in Diabetic Nephropathy. Int. J. Endocrinol. 2018, 2018, 1-13.

7. Kalwat, M. A.; Cobb, M. H. Mechanisms of the amplifying pathway of insulin secretion in the $\beta$ cell. Pharmacol. Ther. 2017, 179, 17-30.

8. Pyne, N. J.; Furman, B. L. Cyclic nucleotide phosphodiesterases in pancreatic islets. Diabetologia 2003, 46, 1179-1189.

9. Ahrén, B. Evidence that autonomic mechanisms contribute to the adaptive increase in insulin secretion during dexamethasone-induced insulin resistance in humans. 2008, 1018-1024.

10. Sherifali, D.; Nerenberg, K.; Pullenayegum, E.; Cheng, J. E.; Gernstein, H. C. The Effect of Oral Antidiabetic Agents on A1C Levels. A systematic review and meta-analysis. Diabetes Care 2010, 33, 1859-1864.

11. Zhao, Z.; Low, Y. S.; Armstrong, N. A.; Ryu, J. H.; Sun, S. A.; Arvanites, A. C.; Hollister-lock, J.; Shah, N. H.; Weir, G. C.; Annes, J. P. Promote $\square$-Cell Replication. Mol Endocrinol 2014, 28, 1682-1697.

12. Ashcroft, F. M.; Rorsman, P. Diabetes mellitus and the $\beta$ cell: The last ten years. Cell 2012, 148, 1160-1171.

13. Lupse, B.; Annamalai, K.; Ibrahim, H.; Kaur, S.; Geravandi, S.; Sarma, B.; Pal, A.; Awal, S.; Joshi, A.; Rafizadeh, S.; et al. Inhibition of PHLPP1/2 phosphatases rescues pancreatic $\beta$-cells in diabetes. Cell Rep. 2021, 36 .

14. Marchetti, P.; Suleiman, M.; De Luca, C.; Baronti, W.; Bosi, E.; Tesi, M.; Marselli, L. A direct look at the dysfunction and pathology of the $\beta$ cells in human type 2 diabetes. Semin. Cell Dev. Biol. 2020, 103, 83-93.

15. Randeria, S. N.; Thomson, G. J. A.; Nell, T. A.; Roberts, T.; Pretorius, E. Inflammatory cytokines in type 2 diabetes mellitus as facilitators of hypercoagulation and abnormal clot formation. Cardiovasc.

Diabetol. 2019, 18, 1-15.

16. Ding, M.; Fang, Q. H.; Cui, Y. T.; Shen, Q. L.; Liu, Q.; Wang, P. H.; Yu, D. M.; Li, C. J. Liraglutide prevents $\beta$-cell apoptosis via inactivation of NOX2 and its related signaling pathway. J. Diabetes Complications 2019, 33, 267-277.

17. Wang, Y.; Li, M.; Yu, X.; He, S.; Wu, X.; Wang, Y. Mulberry leaf flavonoids protect against glucotoxicity-induced INS-1 cell apoptosis. J. Tradit. Chinese Med. 2019, 39, 153-159.

18. Römer, A.; Linn, T.; Petry, S. F. Lipotoxic Impairment of Mitochondrial Function in $\beta$-Cells : A Review. Antioxidants 2021, 10, 1-27.

19. Al-mrabeh, A. $\beta$-Cell Dysfunction, Hepatic Lipid Metabolism, and Cardiovascular Health in Type 2 Diabetes: New Directions of Research and Novel Therapeutic Strategies. Biomedicines 2021, 9, 1-25. 
20. Šrámek, J.; Němcová, V.; Kovář, J. Calcium channel blockers do not protect against saturated fatty acid-induced ER stress and apoptosis in human pancreatic $\beta$-cells. Nutr. Metab. 2021, 18, 1-9.

21. Šrámek, J.; Němcová-fürstová, V.; Kovář, J. Molecular mechanisms of apoptosis induction and its regulation by fatty acids in pancreatic $\beta$-cells. Int. J. Mol. Sci. 2021, 22.

22. Back, S. H.; Kaufman, R. J. Endoplasmic Reticulum Stress and Type 2 Diabetes. Annu Rev Biochem. 2012, 81, 767-793.

23. Al-Nahdi, A. M. T.; John, A.; Haider, R. Cytoprotective Effects of N-Acetylcysteine on StreptozotocinInduced Oxidative Stress and Apoptosis in RIN-5F Pancreatic. Cell Physiol Biochem 2018, 51, 201-216.

24. Hayden, M. R. The Central Role of $\beta$-Cell Dysfunction, Apoptosis and Exploration of Possible Mechanisms. 2020, 1-23.

25. Bruni, A.; Bornstein, S.; Linkermann, A.; Shapiro, A. M. J. Regulated Cell Death Seen through the Lens of Islet Transplantation. Cell Transplant. 2018, 27, 890-901.

26. Cerf, M. E. Beta cell physiological dynamics and dysfunctional transitions in response to islet inflammation in obesity and diabetes. Metabolites 2020, 10, 1-12.

27. Costes, S.; Bertrand, G.; Ravier, M. A. Mechanisms of Beta-Cell Apoptosis in Type 2 Diabetes-Prone Situations and Potential Protection by GLP-1-Based Therapies. Int. J. Mol. Sci. 2021, 22, 5303.

28. Lee, J.; Mellado-gil, J. M.; Bahn, Y. J.; Pathy, S. M.; Zhang, Y. E. Protection from $\beta$-cell apoptosis by inhibition of TGF- $\beta$ / Smad3 signaling. Cell Death Dis. 2020, 11, 1-15.

29. Singh, R.; Letai, A.; Sarosiek, K. Regulation of apoptosis in health and disease: the balancing act of BCL-2 family proteins. Nat Rev Mol Cell Biol. 2019 March 2020, 20, 175-193.

30. Shaheen, A. Effect of the unfolded protein response on ER protein export : a potential new mechanism to relieve ER stress. Cell Stress Chaperones 2018, 23, 797-806.

31. Dlamini, Z.; Mokoena, F.; Hull, R. Abnormalities in alternative splicing in diabetes: Therapeutic targets. J. Mol. Endocrinol. 2017, 59, R93-R107.

32. Uhlemeyer, C.; Muller, N.; Grieß, K.; Wessel, C.; Schlegel, C.; Kuboth, J.; Belgardt, B.-F. ATM and P53 differentially regulate pancreatic beta cell survival in Ins 1E cells. PLoS One 2020, 15, e0237669.

33. Li, X.; Day, N. J.; Feng, S.; Gaffrey, M. J.; Lin, T.; Paurus, V. L.; Monroe, M. E.; Moore, R. J.; Yang, B.; Xian, M.; et al. Redox Biology Mass spectrometry-based direct detection of multiple types of protein thiol modifications in pancreatic beta cells under endoplasmic reticulum stress. Redox Biol. 2021, 46, 102111.

34. Eletto, D.; Chevet, E.; Argon, Y.; Appenzeller-herzog, C. Redox controls UPR to control redox. J. Cell Sci. 2014, 127, 3649-3658.

35. Shrestha, N.; Franco, E. De; Arvan, P.; Cnop, M. Pathological b -Cell Endoplasmic Reticulum Stress in Type 2 Diabetes : Current Evidence. 2021, 12, 1-7.

36. Mustapha, S.; Mohammed, M.; Azemi, A. K.; Yunusa, I.; Shehu, A.; Mustapha, L.; Wada, Y.; Ahmad, M. H.; Ahmad, W. A. N. W.; Rasool, A. H. G.; et al. Potential Roles of Endoplasmic Reticulum Stress and Cellular Proteins Implicated in Diabesity. Oxid Med Cell Longev. 2021, 2021, 1-18.

37. Weir, G. C. Glucolipotoxicity , b -Cells, and Diabetes: The Emperor Has No Clothes. Diabetes 2020, 69, 273-278.

38. Lindenboim, L.; Grozki, D.; Amsalem-Zafran, A. R.; Peña-Blanco, A.; Gundersen, G. G.; Borner, C.; Hodzic, D.; Garcia-Sáez, A. J.; Worman, H. J.; Stein, R. Apoptotic stress induces Bax-dependent, caspase-independent redistribution of LINC complex nesprins. Cell Death Discov. 2020, 6.

39. Lee, H.-C.; Wei, Y.-H. Mitochondrial Role in Life and Death of the Cell Hsin-Chen. J. Biomed. Sci. 2000, 7, 2-15. 
40. Popgeorgiev, N.; Jabbour, L.; Gillet, G. Subcellular localization and dynamics of the Bcl-2 family of proteins. Front. Cell Dev. Biol. 2018, 6, 1-11.

41. Padanilam, B. J. Cell death induced by acute renal injury: A perspective on the contributions of apoptosis and necrosis. Am. J. Physiol. - Ren. Physiol. 2003, 284, 608-627.

42. Johnstone, R. W.; Ruefli, A. A.; Lowe, S. W. Apoptosis: A link between cancer genetics and chemotherapy. Cell 2002, 108, 153-164.

43. Mao, Y.; Xi, L.; Li, Q.; Cai, Z.; Lai, Y.; Zhang, X.; Yu, C. Regulation of cell apoptosis and proliferation in pancreatic cancer through PI3K/Akt pathway via Polo-like kinase 1. Oncol. Rep. 2016, 36, 49-56.

44. Anuradha, R.; Saraswati, M.; Kumar, K. G.; Rani, S. H. Apoptosis of beta cells in diabetes mellitus. DNA Cell Biol. 2014, 33, 743-748.

45. Bhatia, M. Apoptosis versus necrosis in acute pancreatitis. Am. J. Physiol. - Gastrointest. Liver Physiol. 2004, 286, 189-196.

46. Tomita, T. Apoptosis in pancreatic $\beta$-islet cells in Type 2 diabetes. Bosn J Basic Med Sci. 2016, 16, $162-179$.

47. Miani, M.; Elvira, B.; Gurzov, E. N. Sweet Killing in Obesity and Diabetes : The Metabolic Role of the BH3-only Protein BIM. J. Mol. Biol. 2018, 430, 3041-3050.

48. Morris, J. L.; Gillet, G.; Prudent, J.; Popgeorgiev, N. Bcl-2 Family of Proteins in the Control of Mitochondrial Calcium Signalling: An Old Chap with New Roles. Int. J. Mol. Sci. 2021, 22, 3730.

49. Youle, R. J.; Strasser, A. The BCL-2 protein family: opposing activities that mediate cell death. Nat. Rev. Mol. Cell Biol. Vol. 2008, 9, 47-59.

50. Tang, D.; Kang, R.; Berghe, T. Vanden; Vandenabeele, P.; Kroemer, G. The molecular machinery of regulated cell death. Cell Res. 2019, 29, 347-364.

51. Chan, J. Y.; Bensellam, M.; Lin, R. C. Y.; Liang, C.; Lee, K.; Jonas, J. C.; Laybutt, D. R. Transcriptome analysis of islets from diabetes-resistant and diabetes-prone obese mice reveals novel gene regulatory networks involved in beta-cell compensation and failure. FASEB J. 2021, 35, 1-21.

52. Tabas, I.; Ron, D. Integrating the mechanisms of apoptosis induced endoplasmic reticulum stress. Nat Cell Biol. 2011, 13, 184-190.

53. Elmore, S. Apoptosis: A Review of Programmed Cell Death. Toxicol Pathol. 2007 2007, 35, 495-516.

54. Zhang, M.; Harashima, N.; Moritani, T.; Huang, W.; Harada, M. The roles of ROS and caspases in TRAIL-induced apoptosis and necroptosis in human pancreatic cancer cells. PLoS One 2015, 10, 1-15.

55. Criddle, D. N.; Gerasimenko, J. V.; Baumgartner, H. K.; Jaffar, M.; Voronina, S.; Sutton, R.; Petersen, O. H.; Gerasimenko, O. V. Calcium signalling and pancreatic cell death: Apoptosis or necrosis? Cell Death Differ. 2007, 14, 1285-1294.

56. Mareninova, O. A.; Sung, K. F.; Hong, P.; Lugea, A.; Pandol, S. J.; Gukovsky, I.; Gukovskaya, A. S. Cell death in pancreatitis: Caspases protect from necrotizing pancreatitis. J. Biol. Chem. 2006, 281, 3370-3381.

57. Galluzzi, L.; Bravo-San Pedro, J. M.; Vitale, I.; Aaronson, S. A.; Abrams, J. M.; Adam, D.; Alnemri, E. S.; Altucci, L.; Andrews, D.; Annicchiarico-Petruzzelli, M.; et al. Essential versus accessory aspects of cell death: Recommendations of the NCCD 2015. Cell Death Differ. 2015, 22, 58-73.

58. Sharma, A.; Yerra, V. G.; Kumar, A. Emerging role of Hippo signalling in pancreatic biology: YAP re-expression and plausible link to islet cell apoptosis and replication. Biochimie 2017, 133, 56-65.

59. Ardestani, A.; Maedler, K. The Hippo signaling pathway in pancreatic $\beta$-cells: Functions and regulations. Endocr. Rev. 2017, 39, 21-35. 
60. Rausch, V.; Hansen, C. G. The Hippo Pathway, YAP/TAZ, and the Plasma Membrane. Trends Cell Biol. 2020, 30, 32-48.

61. Chang, Y. C.; Wu, J. W.; Wang, C. W.; Jang, A. C. C. Hippo Signaling-Mediated Mechanotransduction in Cell Movement and Cancer Metastasis. Front. Mol. Biosci. 2020, 6, 1-7.

62. Wu, S.; Huang, J.; Dong, J.; Pan, D. hippo encodes a Ste-20 family protein kinase that restricts cell proliferation and promotes apoptosis in conjunction with salvador and warts. Cell 2003, 114, 445-456.

63. Tao, L.; Chen, X.; Zheng, Y.; Wu, Y.; Jiang, X.; You, M.; Li, S.; Hu, F. Chinese Propolis Suppressed Pancreatic Cancer Panc-1 Cells Proliferation and Migration via Hippo-YAP Pathway. Molecules 2021, 26, $1-15$.

64. Avruch, J.; Zhou, D.; Fitamant, J.; Bardeesy, N.; Mou, F.; Barrufet, L. R. Protein Kinases of the Hippo Pathway: Regulation and Substrates. Semin Cell Dev Biol. 2012, 23, 770-784.

65. Saucedo, L. J.; Edgar, B. A. Filling out the Hippo pathway. Nat. Rev. Mol. Cell Biol. 2007, 8, 613-621.

66. Zhao, B.; Li, L.; Lei, Q.; Guan, K. L. The Hippo-YAP pathway in organ size control and tumorigenesis: An updated version. Genes Dev. 2010, 24, 862-874.

67. Fallahi, E.; O’Driscoll, N. A.; Matallanas, D. The MST/Hippo pathway and cell death: A non-canonical affair. Genes (Basel). 2016, 7, 1-31.

68. Matallanas, D.; Romano, D.; Hamilton, G.; Kolch, W.; Neill, O.; Matallanas, D.; Romano, D.; Hamilton, G.; Kolch, W.; Neill, E. O. A Hippo in the ointment: MST signalling beyond the fly. Cell Cycle 2008, 7, 879-884.

69. Dong, J.; Feldmann, G.; Huang, J.; Wu, S.; Zhang, N.; Sarah, A.; Gayyed, M. F.; Anders, R. A.; Maitra, A.; Pan, D. Elucidation of a Universal Size-Control Mechanism in Drosophila and Mammals. Cell 2009, 130, 1120-1133.

70. Maugeri-Saccàa, M.; De Maria, R. The Hippo pathway in normal development and cancer. Pharmacol. Ther. 2018, 186, 60-72.

71. Holden, J. K.; Cunningham, C. N. Targeting the hippo pathway and cancer through the TEAD family of transcription factors. Cancers (Basel). 2018, 10.

72. Misra, J. R.; Irvine, K. D. The hippo signaling network and its biological functions. Annu. Rev. Genet. 2018, 52, 65-87.

73. Zanconato, F.; Cordenonsi, M.; Piccolo, S. YAP / TAZ at the roots of cancer. Cancer Cell. 2018, 29, 783-803.

74. Wu, Y.; Aegerter, P.; Nipper, M.; Ramjit, L.; Liu, J.; Wang, P. Hippo Signaling Pathway in Pancreas Development. Front. Cell Dev. Biol. 2021, 9.

75. Iwasa, H.; Hossain, S.; Hata, Y. Tumor suppressor C - RASSF proteins. Cell. Mol. Life Sci. 2018, 75, 1773-1787.

76. Ardestani, A.; Paroni, F.; Azizi, Z.; Kaur, S.; Khobragade, V. MST1 is a novel regulator of apoptosis in pancreatic beta-cells. Nat. Med. 2014, 20, 385-397.

77. Yuan, T.; Maedler, K.; Ardestani, A. Pancreatic $\beta$-cell rescue in diabetes by targeting Merlin. Expert Rev. Endocrinol. Metab. 2017, 12, 97-99.

78. Liu, J.; Li, J.; Chen, H.; Wang, R.; Li, P.; Miao, Y.; Liu, P. Metformin suppresses proliferation and invasion of drug-resistant breast cancer cells by activation of the Hippo pathway. J. Cell. Mol. Med. 2020, 24, 5786-5796.

79. Yuan, T.; Annamalai, K.; Naik, S.; Lupse, B.; Geravandi, S.; Pal, A.; Dobrowolski, A.; Ghawali, J.; Ruhlandt, M.; Gorrepati, K. D. D.; et al. The Hippo kinase LATS2 impairs pancreatic $\beta$-cell survival in 
diabetes through the mTORC1-autophagy axis. Nat. Commun. 2021, 12, 1-18.

80. Shu, Z.; Gao, Y.; Zhang, G.; Zhou, Y.; Cao, J.; Wan, D.; Zhu, X.; Xiong, W. A functional interaction between Hippo-YAP signalling and SREBPs mediates hepatic steatosis in diabetic mice. J. Cell. Mol. Med. 2019, 23, 3616-3628.

81. Praskova, M.; Xia, F.; Avruch, J. MOBKL1A/MOBKL1B Phosphorylation by MST1 and MST2 Inhibits Cell Proliferation. Curr Biol. 2008, 18, 311-321.

82. Oceandy, D.; Amanda, B.; Ashari, F. Y.; Faizah, Z.; Aziz, M. A.; Sta, N. The Cross-Talk Between the TNF- $\alpha$ and RASSF-Hippo Signalling Pathways. Int. J. Mol. Sci. 2019, 20, 2346.

83. Ni, L.; Li, S.; Yu, J.; Min, J.; Brautigam, C. A.; Tomchick, D. R.; Pan, D.; Luo, X. Structural Basis for Autoactivation of Human Mst2 Kinase and Its Regulation by RASSF5. Structure 2013, 21, 1757-1768.

84. Hwang, E.; Ryu, K.-S.; Paakkonen, K.; Guntert, P.; Cheong, H.-K.; Lim, D.-S.; Lee, J.-O.; Jeon, Y. H.; Cheong, C. Structural insight into dimeric interaction of the SARAH domains from Mst1 and RASSF family proteins in the apoptosis pathway. PNAS 2007, 10, 9236-9241.

85. Li, Y.; Zhou, H.; Li, F.; Chan, S. W.; Lin, Z.; Wei, Z.; Yang, Z.; Guo, F.; Lim, C. J.; Xing, W.; et al. Angiomotin binding-induced activation of Merlin/NF2 in the Hippo pathway. Cell Res. 2015, 25, 801-817.

86. Zhang, N.; Bai, H.; David, K. K.; Dong, J.; Zheng, Y.; Cai, J.; Giovannini, M.; Liu, P.; Anders, R. A.; Pan, D. The Merlin/NF2 Tumor Suppressor Functions through the YAP Oncoprotein to Regulate Tissue Homeostasis in Mammals. Dev. Cell 2010, 19, 27-38.

87. Yuan, T.; Gorrepati, K. D. D.; Maedler, K.; Ardestani, A. Loss of Merlin/NF2 protects pancreatic $\beta$-cells from apoptosis by inhibiting LATS2. Cell Death Dis. 2016, 7, 10-11.

88. Yin, F.; Yu, J.; Zheng, Y.; Chen, Q.; Zhang, N.; Pan, D. Spatial organization of Hippo signaling at the plasma membrane mediated by the tumor suppressor Merlin/NF2. Cell. 2013, 154, 1-7.

89. Thompson, B. J.; Sahai, E. MST kinases in development and disease. J. Cell Biol. Vol. 2015, 210, $871-882$.

90. Zhao, B.; Wei, X.; Li, W.; Udan, R. S.; Yang, Q.; Kim, J.; Xie, J.; Ikenoue, T.; Yu, J.; Li, L.; et al. Inactivation of YAP oncoprotein by the Hippo pathway is involved in cell contact inhibition and tissue growth control. GENES Dev. 2007, 21, 2747-2761.

91. Yu, F.-X.; Zhao, B.; Guan, K.-L. Hippo Pathway in Organ Size Control, Tissue Homeostasis, and Cancer. Physiol. Behav. 2015, 163, 811-828.

92. Pan, D. The Hippo Signaling Pathway in Development and Cancer. Dev Cell. 2010, 19, 491-505.

93. Pombo, C. M.; Iglesias, C.; Sartages, M.; Zalvide, J. B. MST Kinases and Metabolism. Endocrinology 2019, 160, 1111-1118.

94. Praskova, M.; Khoklatchev, A.; Ortiz-vega, S.; Avruch, J. Regulation of the MST1 kinase by autophosphorylation, by the growth inhibitory proteins, RASSF1 and NORE1, and by Ras. Biochem. J. 2004, 462, 453-462.

95. Galan, J. A.; Avruch, J. MST1/MST2 Protein Kinases: Regulation and Physiologic Roles. Biochemistry 2016, 55, 5507-5519.

96. Creasy, C. L.; Ambrose, D. M.; Chernoff, J. The Ste20-like Protein Kinase, Mst1 , Dimerizes and Contains an Inhibitory Domain *. J. Biol. Chem. 1996, 271, 21049-21053.

97. Bitra, A.; Sistla, S.; Mariam, J.; Malvi, H.; Anand, R. Rassf Proteins as Modulators of Mst1 Kinase Activity. Nat. Publ. Gr. 2017, 1-11.

98. Lee, K. K.; Ohyama, T.; Yajima, N.; Tsubuki, S.; Yonehara, S. MST, a Physiological Caspase Substrate, Highly Sensitizes Apoptosis Both Upstream and Downstream of Caspase Activation. J. Biol. Chem. 2001, 
276, 19276-19285.

99. Ura, S.; Masuyama, N.; Graves, J. D.; Gotoh, Y. Caspase cleavage of MST1 promotes nuclear translocation and chromatin condensation. PNAS 2001, 98, 10148-10153.

100. Graves, J. D.; Gotoh, Y.; Draves, K. E.; Ambrose, D.; Han, D. K. M.; Wright, M.; Chernoff, J.; Clark, E. A.; Krebs, E. G. Caspase-mediated activation and induction of apoptosis by the mammalian Ste20-like kinase Mst1. EMBO J. 1998, 17, 2224-2234.

101. Anand, R.; Kim, A.-Y.; Brent, M.; Marmorstein, R. Biochemical Analysis of MST1 Kinase: Elucidation of a C-Terminal Regulatory Region. Biochemistry 2008, 47, 6719-6726.

102. Ardestani, A.; Maedler, K. MST1 : a promising therapeutic target to restore functional beta cell mass in diabetes. Diabetologia 2016, 59, 1843-1849.

103. Rahmani, M.; Anderson, A.; Habibi, J. R.; Crabtree, T. R.; Mayo, M.; Harada, H.; Ferreira-Gonzalez, A.; Dent, P.; Grant, S. The BH3-only protein Bim plays a critical role in leukemia cell death triggered by concomitant inhibition of the PI3K/Akt and MEK/ERK1/2 pathways. Blood 2009, 114, 4507-4516.

104. Lei, K.; Davis, R. J. JNK phosphorylation of Bim-related members of the Bcl 2 family induces Bax-dependent apoptosis. Proc. Natl. Acad. Sci. U. S. A. 2003, 100, 2432-2437.

105. Rudel, T.; Bokoch, G. M. Membrane and Morphological Changes in Apoptotic Cells Regulated by Caspase-Mediated Activation of PAK2. Science (80-. ). 1997, 276, 1571-1575.

106. Song, H.; Kingston, K.; Topol, L.; Yun, K.; Hu, J.; Garrett, L.; Chen, Y.; Park, O. Mammalian Mst1 and Mst2 kinases play essential roles in organ size control and tumor suppression. PNAS 2010, 107, 1431-1436.

107. Zhou, D.; Conrad, C.; Xia, F.; Park, J.; Payer, B.; Lauwers, G. Y.; Thasler, W.; Lee, J. T.; Avruch, J.; Bardeesy, N. Mst1 and Mst2 maintain hepatocyte quiescence and suppress the development of hepatocellular carcinoma through inactivation of the Yap1 oncogene. Cancer Cell. 2009, 16, 425-438.

108. Yun, H. J.; Yoon, J.; Lee, J. K.; Noh, K.; Yoon, K.; Oh, S. P.; Oh, H. J.; Chae, J. S.; Hwang, S. G.; Kim, E. H.; et al. Daxx mediates activation-induced cell death in microglia by triggering MST1 signalling. EMBO J. 2011, 30, 2465-2476.

109. Cinar, B.; Fang, P. K.; Lutchman, M.; Di Vizio, D.; Adam, R. M.; Pavlova, N.; Rubin, M. A.; Yelick, P. C.; Freeman, M. R. The pro-apoptotic kinase Mst1 and its caspase cleavage products are direct inhibitors of Akt1. EMBO J. 2007, 26, 4523-4534.

110. Ardestani, A.; Li, S.; Annamalai, K.; Lupse, B.; Geravandi, S.; Dobrowolski, A.; Yu, S.; Zhu, S.; Baguley, T. D.; Surakattula, M.; et al. Neratinib protects pancreatic beta cells in diabetes. Nat. Commun. 2019, 10, 1-17.

111. Ardestani, A.; Tremblay, M. S.; Shen, W.; Maedler, K. Neratinib is an MST1 inhibitor and restores pancreatic $\beta$-cells in diabetes. Cell Death Discov. 2019, 5, 10-12.

112. Ni, L.; Li, S.; Yu, J.; Min, J.; Brautigam, C. A.; Tomchick, D. R.; Pan, D.; Luo, X. Structural Basis for Autoactivation of Human Mst2 Kinase and Its Regulation by RASSF5. Structure. 2013, 21, 1757-1768.

113. Ni, L.; Zheng, Y.; Hara, M.; Pan, D.; Luo, X. Structural basis for Mob1-dependent activation of the core Mst - Lats kinase cascade in Hippo signaling. GENES Dev. 2015, 29, 1416-1431.

114. Cohen, P.; Goedert, M. GSK3 inhibitors: Development and therapeutic potential. Nat. Rev. Drug Discov. 2004, 3, 479-487.

115. Cohen, P.; Cross, D.; Jänne, P. A. Kinase drug discovery 20 years after imatinib: progress and future directions. Nat. Rev. Drug Discov. 2021, 20, 551-569.

116. Agostino, N. M.; Chinchilli, V. M.; Lynch, C. J.; Koszyk-Szewczyk, A.; Gingrich, R.; Sivik, J.; 
Drabick, J. J. Effect of the tyrosine kinase inhibitors (sunitinib, sorafenib, dasatinib, and imatinib) on blood glucose levels in diabetic and nondiabetic patients in general clinical practice. J. Oncol. Pharm. Pract. 2011, 17, 197-202.

117. Ono, K.; Suzushima, H.; Watanabe, Y.; Kikukawa, Y.; Shimomura, T.; Furukawa, N.; Kawaguchi, T.; Araki, E. Rapid amelioration of hyperglycemia facilitated by dasatinib in a chronic myeloid leukemia patient with type 2 diabetes mellitus. Intern. Med. 2012, 51, 2763-2766.

118. Gómez-Sámano, M. Á.; Baquerizo-Burgos, J. E.; Coronel, M. F. C.; Wong-Campoverde, B. D.; Villanueva-Martinez, F.; Molina-Botello, D.; Avila-Rojo, J. A.; Palacios-Báez, L.; Cuevas-Ramos, D.; Gomez-Perez, F. J.; et al. Effect of imatinib on plasma glucose concentration in subjects with chronic myeloid leukemia and gastrointestinal stromal tumor 11 Medical and Health Sciences 1103 Clinical Sciences. BMC Endocr. Disord. 2018, 18, 1-8.

119. Oh, J. J.; Hong, S. K.; Joo, Y. M.; Lee, B. K.; Min, S. H.; Lee, S.; Byun, S. S.; Lee, S. E. Impact of sunitinib treatment on blood glucose levels in patients with metastatic renal cell carcinoma. Jpn. J. Clin. Oncol. 2012, 42, 314-317.

120. Brooks, M. B. Erlotinib and Gefitinib, EpidermalGrowthFactor Receptor Kinase Inhibitors,MayTreat Non-Cancer-RelatedTumorNecrosis Factor-alfa MediatedInflammatory Diseases. Oncologist 2013, 18, e3-e5.

121. Klil-Drori, A. J.; Azoulay, L.; Pollak, M. N. Cancer, obesity, diabetes, and antidiabetic drugs: Is the fog clearing? Nat. Rev. Clin. Oncol. 2016, 14, 85-99.

122. Fountas, A.; Diamantopoulos, L. N.; Tsatsoulis, A. Tyrosine Kinase Inhibitors and Diabetes: A Novel Treatment Paradigm? Trends Endocrinol. Metab. 2015, 26, 643-656.

123. Huda, M. S. B.; Amiel, S. A.; Ross, P.; Aylwin, S. J. B. Tyrosine kinase inhibitor sunitinib allows insulin independence in long-standing type 1 diabetes. Diabetes Care 2014, 37, 87-88.

124. Hägerkyist, R.; Jansson, L.; Welsh, N. Imatinib mesylate improves insulin sensitivity and glucose disposal rates in rats fed a high-fat diet. Clin. Sci. 2008, 114, 65-71.

125. Box, C. V. J.; Sandhu, A. K.; Turaihi, A. H.; Xiaoké, P.; Dallinga-Thie, G.; Aman, J.; Eringa, E. C. Effects of imatinib on vascular insulin sensitivity and free fatty acid transport in early weight gain. PLoS One 2021, 16, 1-19.

126. Han, M. S.; Chung, K. W.; Cheon, H. G.; Rhee, S. D.; Yoon, C. H.; Lee, M. K.; Kim, K. W.; Lee, M. S. Imatinib mesylate reduces endoplasmic reticulum stress and induces remission of Diabetes in $\mathrm{db} / \mathrm{db}$ mice. Diabetes 2009, 58, 329-336.

127. Kubiatowski, T. Neratinib in extended adjuvant therapy for HER2-positive early breast cancer. Oncol. Clin. Pract. 2020, 16, 201-205.

128. Cedrych, E.; Cedrych, I. Neratinib in adjuvant treatment of patients with HER2-positive breast cancer — Less is more? Oncol. Clin. Pract. 2019, 15, 111-114.

129. Lüftner, D.; Tesch, H.; Schmidt, M.; Hartkopf, A. D.; Streicher, S.; Resch, A.; Genovese, L.; Rosé, C.; Valenti, R.; Harbeck, N. Neratinib as extended adjuvant therapy in patients with copositive early breast cancer: German health technology assessment-driven analyses from the ExteNET study. Eur. J. Cancer 2021, 150, 268-277.

130. Faizah, Z.; Amanda, B.; Ashari, F. Y.; Triastuti, E.; Oxtoby, R.; Rahaju, A. S.; Aziz, M. A.; Lusida, M. I.; Oceandy, D. Treatment with mammalian Ste-20-like kinase 1/2 (MST1/2) inhibitor XMU-MP-1 improves glucose tolerance in streptozotocin-induced diabetes mice. Molecules 2020, 25.

131. Fan, F.; He, Z.; Kong, L.; Chen, Q.; Yuan, Q.; Zhang, S. Pharmacological targeting of kinases MST1 
and MST2 augments tissue repair and regeneration. Sci. Transl. Med. 2016, 8, 352ra108-352ra108. 132. Arthur, E.; Kittur, F. S.; Lin, Y.; Hung, C.; Sane, D. C. Plant-Produced Asialo-Erythropoietin Restores Pancreatic Beta-Cell Function by Suppressing Mammalian Sterile-20-like Kinase ( MST1 ) and Caspase-3 Activation. Front. Pharmacol. 2017, 8, 1-10.

133. Emerson, J. P.; Cabelli, D. E.; Donald, M.; Erbayraktar, S.; Grasso, G.; Sfac-, A.; Xie, Q.; Coleman, T.; Kreilgaard, M.; Sager, T.; et al. Asialoerythropoietin is a nonerythropoietic cytokine with broad neuroprotective activity in vivo. PNAS 2003, 100, 6741-6746.

134. Rey, F.; Ottolenghi, S.; Giallongo, T.; Balsari, A.; Martinelli, C.; Rey, R.; Allevi, R.; Maria, A.; Giulio, D.; Zuccotti, G. V.; et al. Mitochondrial Metabolism as Target of the Neuroprotective Role of Erythropoietin in Parkinson' s Disease. Antioxidants 2021, 10, 1-21.

135. Yu, F.-X.; Zhao, B.; Guan, K.-L. Hippo Pathway in Organ Size Control, Tissue Homeostasis, and Cancer. Cell 2015, 163, 811-828.

136. Justice, R. W.; Zilian, O.; Woods, D. F.; Noll, M.; Bryant, P. J. The Drosophila tumor suppressor gene warts encodes a homolog of human myotonic dystrophy kinase and is required for the control of cell shape and proliferation. Genes Dev. 1995, 9, 534-546.

137. Furth, N.; Aylon, Y. The LATS1 and LATS2 tumor suppressors: Beyond the hippo pathway. Cell Death Differ. 2017, 24, 1488-1501.

138. Hori, T.; Takaori-Kondo, A.; Kamikubo, Y.; Uchiyama, T. Molecular cloning of a novel human protein kinase, kpm, that is homologous to warts/lats, a Drosophila tumor suppressor. Oncogene 2000, 19, 3101-3109.

139. Yabuta, N.; Mukai, S.; Okamoto, A.; Okuzaki, D.; Suzuki, H.; Torigata, K.; Yoshida, K.; Okada, N.; Miura, D.; Ito, A.; et al. N-terminal truncation of Lats1 causes abnormal cell growth control and chromosomal instability. J. Cell Sci. 2013, 126, 508-519.

140. Kim, M.; Kim, M.; Park, S.; Lee, C.; Lim, D. Role of Angiomotin-like 2 mono-ubiquitination on YAP inhibition. EMBO Rep. 2016, 17, 64-78.

141. Sudol, M. Newcomers to the WW domain-mediated network of the hippo tumor suppressor pathway. Genes and Cancer 2010, 1, 1115-1118.

142. Visser, S.; Yang, X. LATS tumor suppressor: A new governor of cellular homeostasis. Cell Cycle 2010, 9, 3892-3903.

143. Zeng, Q.; Hong, W. The Emerging Role of the Hippo Pathway in Cell Contact Inhibition, Organ Size Control, and Cancer Development in Mammals. Cancer Cell 2008, 13, 188-192.

144. Xia, H.; Qi, H.; Li, Y.; Pei, J.; Barton, J.; Blackstad, M.; Xu, T.; Tao, W. LATS1 tumor suppressor regulates G2/M transition and apoptosis. Oncogene 2002, 21, 1233-1241.

145. Petrilli, A. M.; Fernández-Valle, C. Role of Merlin/NF2 inactivation in tumor biology. Oncogene 2016, 35, 537-548.

146. Ke, H.; Pei, J.; Ni, Z.; Xia, H.; Qi, H.; Woods, T.; Kelekar, A.; Tao, W. Putative tumor suppressor Lats2 induces apoptosis through downregula- tion of Bcl-2 and Bcl-x L. Exp. Cell Res. 2004, 298, 329-338.

147. Aylon, Y.; Ofir-rosenfeld, Y.; Yabuta, N.; Lapi, E.; Nojima, H.; Lu, X.; Oren, M. The Lats2 tumor suppressor augments p53-mediated apoptosis by promoting the nuclear proapoptotic function of ASPP1.

2010, 2420-2429.

148. Shao, D.; Zhai, P.; Re, D. P. Del; Sciarretta, S.; Yabuta, N.; Nojima, H.; Lim, D.; Pan, D.; Sadoshima, J.; Biomedical, R.; et al. A Functional Interaction between Hippo-YAP Signaling and FoxO1 Mediates the Oxidative Stress Response. Nat Commun 2014, 5, 3315. 
149. Guillén, C.; Benito, M. MTORC1 overactivation as a key aging factor in the progression to type 2 diabetes mellitus. Front. Endocrinol. (Lausanne). 2018, 9, 1-7.

150. Zou, Z.; Tao, T.; Li, H.; Zhu, X. mTOR signaling pathway and mTOR inhibitors in cancer: progress and challenges. Cell Biosci. 2020, 10, 31.

151. Bu, H.; Liu, D.; Zhang, G.; Chen, L.; Song, Z. AMPK / mTOR / ULK1 Axis-Mediated Pathway Participates in Apoptosis and Autophagy Induction by Oridonin in Colon Cancer DLD-1. Onco. Targets. Ther. 2020, 13, 8533-8545.

152. Kastan, N.; Gnedeva, K.; Alisch, T.; Petelski, A. A.; Huggins, D. J.; Chiaravalli, J.; Aharanov, A.; Shakked, A.; Tzahor, E.; Nagiel, A.; et al. Small-molecule inhibition of Lats kinases may promote Yap-dependent proliferation in postmitotic mammalian tissues. Nat. Commun. 2021, 12, 3100.

153. Property, W. I.; Bureau, I.; Date, I. P.; Number, I. P. Wo 2018/198077 a2 (51) 2018.

154. Calses, P. C.; Crawford, J. J.; Lill, J. R.; Dey, A. Hippo Pathway in Cancer: Aberrant Regulation and Therapeutic Opportunities. Trends in Cancer 2019, 5, 297-307.

155. Liu, Y.; Palanivel, R.; Rai, E.; Park, M.; Gabor, T. V; Scheid, M. P.; Xu, A.; Sweeney, G. Adiponectin Stimulates Autophagy and Reduces Oxidative Stress to Enhance Insulin Sensitivity During High- Fat Diet Feeding in Mice. Diabetes 2015, 64, 36-48.

156. Lim, Y.; Lim, H.; Hur, K. Y.; Quan, W.; Lee, H.; Cheon, H.; Ryu, D.; Koo, S.; Kim, H. L.; Kim, J.; et al. progression from obesity to diabetes. Nat. Commun. 2014, 5, 4934.

157. Liu, Y.; Shi, S.; Gu, Z.; Du, Y. Impaired autophagic function in rat islets with aging. Age (Omaha). 2013, 35, 1531-1544.

158. He, C.; Bassik, M. C.; Moresi, V.; Sun, K.; Wei, Y.; Zou, Z.; An, Z.; Loh, J.; Fisher, J.; Sun, Q.; et al. Exercise-induced BCL2-regulated autophagy is required for muscle glucose homeostasis. Nature 2012, 481, 511-515.

159. Ardestani, A.; Lupse, B.; Kido, Y.; Leibowitz, G.; Maedler, K. mTORC1 Signaling: A Double-Edged Sword in Diabetic $\beta$ Cells. Cell Metab. 2018, 27, 314-331.

160. Komuro, A.; Nagai, M.; Navin, N. E.; Sudol, M. WW domain-containing protein YAP associates with ErbB-4 and acts as a co-transcriptional activator for the carboxyl-terminal fragment of ErbB-4 that translocates to the nucleus. J. Biol. Chem. 2003, 278, 33334-33341.

161. Sudol, M.; Shields, D. C.; Farooq, A. Structures of YAP protein domains reveal promising targets for development of new cancer drugs. Semin Cell Dev Biol. 2012, 23, 827-833.

162. Ma, R.; Ren, J. M.; Li, P.; Zhou, Y. J.; Zhou, M. K.; Hu, Z.; Xiao, X. Y. Activated YAP causes renal damage of type 2 diabetic nephropathy. Eur. Rev. Med. Pharmacol. Sci. 2019, 23, 755-763.

163. Low, B. C.; Pan, C. Q.; Shivashankar, G. V.; Bershadsky, A.; Sudol, M.; Sheetz, M. YAP/TAZ as mechanosensors and mechanotransducers in regulating organ size and tumor growth. FEBS Lett. 2014, 588, 2663-2670.

164. Plouffe, S. W.; Lin, K. C.; Moore, J. L.; Tan, F. E.; Ma, S.; Ye, Z.; Qiu, Y.; Ren, B.; Guan, K. L. The Hippo pathway effector proteins YAP and TAZ have both distinct and overlapping functions in the cell. $J$. Biol. Chem. 2018, 293, 11230-11240.

165. Xie, W.; Xiao, W.; Tang, K.; Zhang, L.; Li, Y. Yes-Associated Protein 1: Role and Treatment Prospects in Orthopedic Degenerative Diseases. Front. Cell Dev. Biol. 2020, 8, 1-13.

166. Hansen, C. G.; Moroishi, T.; Guan, K.-L. YAP and TAZ: a nexus for Hippo signaling and beyond. Trends Cell Biol. 2015 2015, 25, 499-513.

167. Mo, J.-S.; Meng, Z.; Kim, Y. C.; Park, H. W.; Hansen, C. G.; Kim, S.; Lim, D.-S.; Guan, K.-L. Cellular 
energy stress induces AMPK-mediated regulation of YAP and the Hippo pathway. Physiol. Behav. 2015, 17, $500-510$.

168. Watt, K. I.; Harvey, K. F.; Gregorevic, P. Regulation of tissue growth by the mammalian Hippo signaling pathway. Front. Physiol. 2017, 8.

169. Wu, Z.; Guan, K.-L. Hippo Signaling in Embryogenesis and Development. Trends Biochem Sci 2021, 46, 51-63.

170. Heng, B. C.; Zhang, X.; Aubel, D.; Bai, Y.; Li, X.; Wei, Y.; Fussenegger, M.; Deng, X. Role of YAP/TAZ in Cell Lineage Fate Determination and Related Signaling Pathways. Front. Cell Dev. Biol. 2020, $8,1-23$.

171. Li, Y.; Wang, J.; Zhong, W. Regulation and mechanism of YAP/TAZ in the mechanical microenvironment of stem cells (Review). Mol. Med. Rep. 2021, 24, 1-11.

172. Ortillon, J.; Le Bail, J. C.; Villard, E.; Léger, B.; Poirier, B.; Girardot, C.; Beeske, S.; Ledein, L.; Blanchard, V.; Brieu, P.; et al. High Glucose Activates YAP Signaling to Promote Vascular Inflammation. Front. Physiol. 2021, 12, 1-10.

173. Zhang, X.; Zhao, H.; Li, Y.; Xia, D.; Yang, L.; Ma, Y.; Li, H. The role of YAP/TAZ activity in cancer metabolic reprogramming. Mol. Cancer 2018, 17, 1-10.

174. Rozengurt, E.; Sinnett-Smith, J.; Eibl, G. Yes-associated protein (YAP) in pancreatic cancer: at the epicenter of a targetable signaling network associated with patient survival. Signal Transduct. Target. Ther. 2018, 3, 1-10.

175. Meng, Z.; Moroishi, T.; Guan, K. Mechanisms of Hippo pathway regulation. GENES Dev. 2016, 30, $1-17$.

176. Zheng, Y.; Pan, D. The Hippo Signaling Pathway in Development and Disease. Dev. Cell 2019, 50, 264-282.

177. Ikeda, S.; Mukai, R.; Mizushima, W.; Zhai, P.; Oka, S.; Nakamura, M.; Re, D. P. Del; Sciarretta, S.; Hsu, C.-P.; Shimokawa, H.; et al. Yes-Associated Protein (YAP) Facilitates Pressure Overload - Induced Dysfunction in the Diabetic Heart. J Am Coll Cardiol Basic Trans Sci. 2019, 4, 611-622.

178. Sayedyahossein, S.; Hedman, A. C.; Sacks, D. B. Insulin suppresses transcriptional activity of yes-associated protein in insulin target cells. Mol. Biol. Cell 2020, 31, 131-141.

179. Yuan, T.; Rafizadeh, S.; Azizi, Z.; Lupse, B.; Gorrepati, K. D. D.; Awal, S.; Oberholzer, J.; Maedler, K.; Ardestani, A. Proproliferative and antiapoptotic action of exogenously introduced YAP in pancreatic $\beta$ cells. JCI Insight 2016, 1.

180. Zhou, R.; Wu, Q.; Wang, M.; Irani, S.; Li, X.; Zhang, Q.; Meng, F.; Liu, S.; Zhang, F.; Wu, L.; et al. The protein phosphatase PPM1A dephosphorylates and activates YAP to govern mammalian intestinal and liver regeneration. PLoS Biol. 2021, 19, 1-30.

181. Moroishi, T.; Park, H. W.; Qin, B.; Chen, Q.; Meng, Z.; Plouffe, S. W.; Taniguchi, K.; Yu, F. X.; Karin, M.; Pan, D.; et al. A YAP/TAZ-induced feedback mechanism regulates Hippo pathway homeostasis. Genes Dev. 2015, 29, 1271-1284.

182. Panciera, T.; Azzolin, L.; Cordenonsi, M.; Piccolo, S. Mechanobiology of YAP and TAZ in physiology and disease. Nat. Rev. Mol. Cell Biol. 2017, 18, 758-770.

183. Piccolo, S.; Dupont, S.; Cordenonsi, M. The biology of YAP/TAZ: Hippo signaling and beyond. Physiol. Rev. 2014, 94, 1287-1312.

184. Mugahid, D.; Kalocsay, M.; Liu, X.; Gruver, J. S.; Peshkin, L.; Kirschner, M. W. YAP regulates cell size and growth dynamics via non-cell autonomous mediators. Elife 2020, 9, 1-20. 
185. Misfeldt, A. A.; Costa, R. H.; Gannon, M. $\beta$-cell proliferation, but not neogenesis, following $60 \%$ partial pancreatectomy is impaired in the absence of FoxM1. Diabetes 2008, 57, 3069-3077.

186. Hotta, M.; Tashiro, F.; Ikegami, H.; Niwa, H.; Ogihara, T.; Yodoi, J.; Miyazaki, J. I. Pancreatic $\beta$ cell-specific expression of thioredoxin, an antioxidative and antiapoptotic protein, prevents autoimmune and streptozotocin-induced diabetes. J. Exp. Med. 1998, 188, 1445-1451.

187. Yamamoto, M.; Yamato, E.; Shu-Ichi, T.; Tashiro, F.; Ikegami, H.; Yodoi, J.; and Jun-Ichi Miyazaki Transgenic Expression of Antioxidant Protein Thioredoxin in Pancreatic $\beta$ Cells Prevents Progression of Type 2 Diabetes Mellitus. Antioxid. redox Signal. 2007, 10. 